\title{
Tail Asymptotics for a Discrete-Time Priority Preemptive Queueing System
}

\author{
Chen Xu \\ School of Mathematics and Statistics \\ Carleton University \\ 1125 Colonel By Drive \\ Ottawa, Ontario \\ Canada K1S 5B6
}

(C)Chen Xu 2010

December 22, 2010 
Library and Archives

Canada

Published Heritage

Branch

395 Wellington Street Ottawa ON K1A ON4 Canada
Bibliothèque et

Archives Canada

Direction du

Patrimoine de l'édition

395 , rue Wellington Ottawa ON K1A ON4

Canada

Your file Votre référence

ISBN: 978-0-494-79583-5

Our file Notre référence

ISBN: 978-0-494-79583-5

NOTICE:

The author has granted a nonexclusive license allowing Library and Archives Canada to reproduce, publish, archive, preserve, conserve, communicate to the public by telecommunication or on the Internet, loan, distribute and sell theses worldwide, for commercial or noncommercial purposes, in microform, paper, electronic and/or any other formats.

The author retains copyright ownership and moral rights in this thesis. Neither the thesis nor substantial extracts from it may be printed or otherwise reproduced without the author's permission.
AVIS:

L'auteur a accordé une licence non exclusive permettant à la Bibliothèque et Archives Canada de reproduire, publier, archiver, sauvegarder, conserver, transmettre au public par télécommunication ou par l'Internet, prêter, distribuer et vendre des thèses partout dans le monde, à des fins commerciales ou autres, sur support microforme, papier, électronique et/ou autres formats.

L'auteur conserve la propriété du droit d'auteur et des droits moraux qui protège cette thèse. $\mathrm{Ni}$ la thèse ni des extraits substantiels de celle-ci ne doivent être imprimés ou autrement reproduits sans son autorisation.
In compliance with the Canadian Privacy Act some supporting forms may have been removed from this thesis.

While these forms may be included in the document page count, their removal does not represent any loss of content from the thesis.
Conformément à la loi canadienne sur la protection de la vie privée, quelques formulaires secondaires ont été enlevés de cette thèse.

Bien que ces formulaires aient inclus dans la pagination, il n'y aura aucun contenu manquant. 


\begin{abstract}
In this thesis, we consider a discrete-time preemptive priority queue with a single server and two types of customers, which is a counterpart model to the classical continuous-time priority queue system. The two classes of customers arrive at the system independently with a different arrival probability and are being served independently with the same service time probability. With the preemptive rule, a low-priority customer service is interrupted by an arrival of a high-priority customer to the system. The service of the interrupted low-priority customer is restarted when the last high-priority customer has completed its service in the queue. We focus on the characterization of exact tail asymptotic behaviour for the joint stationary distribution. The generating function method is used for this analysis. Our main contributions in this research include: (1) a detailed expression of the generating function of the system and analysis of its key kernel function; (2) an explicit determination of the exact tail asymptotics along the high-priority direction; and (3) the identification of the light-tailed regions for the three different types of exact tail asymptotics along the low-priority direction: exact geometric, geometric multiplied by a power function with power $-1 / 2$ or $-3 / 2$.
\end{abstract}




\section{Acknowledgements}

I am sincerely grateful to all those who gave me the possibility to complete this thesis. I am deeply indebted to my supervisors Dr. Yiqiang Zhao from Carleton University and Dr. Hui Li from Mount Saint Vincent University for their training, advice, and stimulating encouragement. I appreciate Professor Zhao's helpful guidance throughout the research work and for taking me as a student while he was already carrying a heavy workload, including his responsibilities as the director of the School of Mathematics and Statistics. I would like to express my gratitude to Professor Li for her grateful help on my research and graciously taking the time to review and correct my work. I am grateful.

My thanks also go to Mr. Eric Preston, P. Eng. of Hypernetics who supported me by reviewing the final version of this thesis for English style and grammar. I am grateful for his help, suggestions and corrections.

Finally I would like to give my special thanks to my family for motivating me to work hard and encouraging me when I am discouraged. Without their patience and love, this thesis would not have come to fruition. 


\section{Contents}

$\begin{array}{ll}\text { Abstract } & \text { i }\end{array}$

Acknowledgements ii

1 Introduction 1

1.1 Motivations and background ................. 1

1.2 Contributions ........................ 4

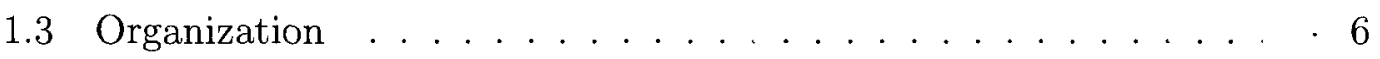

2 Priority Queues - Continuous-time or Discrete-time 8

2.1 Continuous-time preemptie priority queue . . . . . . . . 9

2.2 Discrete-time preemptive priority queue . . . . . . . . . . 13

3 Generating Functions and the Kernel $\quad 18$

3.1 Fundamental form .................... 18

3.2 Key kernel . . . . . . . . . . . . . . . . 26

3.3 Generating functions $\varphi_{j}(x)$ and $\psi_{0}(y) \ldots \ldots . . . . . . .31$

4 Analysis of Singularities 41

5 Exact Tail Asymptotics for the Low-Priority Queue 45 
5.1 Exact Tail Asymptotics of $\pi_{0, j} \ldots \ldots \ldots$. . . . . . . . 45

5.2 Exact Tail Asymptotics of $\pi_{i, \jmath}$ for fixed $i \ldots \ldots$. . . . . . 53

6 Exact Tail Asymptotics for the High-Priority Queue 62

$\begin{array}{lll}7 & \text { Conclusion } & 66\end{array}$ 


\section{List of Figures}

2.1 Transitions for the continuous-time priority queueing system . . . 10

2.2 Time instances of arrivals, departures and observations . . . . . . 14

2.3 Transitions for the discrete-time priority queueing system . . . . 16 


\section{Chapter 1}

\section{Introduction}

\subsection{Motivations and background}

In our everyday life, queueing is an aspect we would meet at every step. For example, when you are in a waiting line at a bank, you are involved in a queue. The study of queueing problem is also motivated by a large number of applications in the field of telecommunications and industrial engineering. For instance, a system involving a large amount of computers and routers, in a communication network, can be viewed as a queueing network. Study of queueing theories provides frameworks for understanding and designing such networks.

In the emergency room or a police call center, customers with different conditions usually require asymmetric treatments. For example, in the emergency room, a severe patient would require immediate medical treatment over a non-severe patient. These kinds of problems motivate the study of priority queues. Furthermore in the ER example, if a severe patient arrives while a non-severe patient is receiving medical treatment, the treatment of the non-severe patient might possibly be interrupted immediately and resumed after the treatment of the last severe 
patient has been completed. This kind of situation in real life is a typical example of a preemptive priority queueing model.

In general, a queueing phenomenon occurs when a number of customers or jobs need to be served by a shared facility. The scarcity of resources in real life causes the study of queueing theories to receive considerable attention in the literature as it can improve service efficiency and is useful for resource management.

The performance of a queue can be characterized by its stationary distribution, which is our focus in queueing studies. However, there is no explicit solution for this stationary distribution when the system is complex, such as the model considered in this thesis. This motivates the study of exact tail asymptotic behaviour of the stationary distribution since it often leads to the performance bounds, approximations and properties of the queueing model. For all these reasons, we can see that analysis on exact tail asymptotics for the stationary distribution is very important and useful in queueing studies.

The classical priority queueing system with one server and two classes of customers is a model in continuous time. This kind of model has been well studied by several researchers. Miller in [36] suggested an efficient computational scheme based on a special structure of the rate matrix. Since then other researchers have extended his work to more general types of priority queueing models. Some of the references include: Gail, Hantler, Taylor [19, 20], Kao and Narayanan [27], Takine [47], Alfa [2], Isotupa and Stanford [25], Alfa, Liu and He [3], Sleptchenko, Adan and van Houtum [45], Drekic and Woolford [8], Zhao et al. [49], Maertens, Walraevens, and Bruneel [32] .

In the literature, there are several approaches on analysis of tail asymptotic behaviour. Each method has advantages in some aspects. Probability generating function method or simply called generating function method is a classical ap- 
proach. It has advantages that the coefficients can be determined explicitly besides the decay rate. In the double quasi-birth-and death (QBD) process and the random walk in the quarter plane, the generating function approach can be applied in principle based on the theory of Riemann surfaces and algebraic ideas. Some reference in this method include Malyshev [33,34], Flatto and McKean [14], Fayolle and Iasnogorodski [9], Fayolle, King and Mitrani [11], Cohen and Boxma [7], Flatto and Hahn [13], Flatto [12], Fayolle, Iasnorgorodski and Malyshev [10], Wright [48], Kurkova and Suhov [29], Morrison [43], and recently Guillemin and van Leeuwarden [21].

Theorems based on large deviation is another approach with the advantage that it is helpful for the analysis of more general processes. Borovkov and Mogul'skii [5] provided properties on tail asymptotics along an arbitrary direction but not including the two coordinate directions while McDonald [35], Foley, McDonald $[16,17,18]$ and Adan, Folye, MacDonald [1] provided the solution to coordinate directions.

For analysis of exact geometric decay along a coordinate direction, the matrix analytic method is an efficient method. It can also provide conditions on non-exact geometric tail asymptotics. Some references in this method include Takahashi, Fujimoto and Makimoto [46], Haque [22], Miyazawa [38], Miyazawa and Zhao [42], Kroese, Scheinhardt and Taylor [28], Haque, Liu and Zhao [23], Motyer and Taylor [44], Li, Miyazawa and Zhao [30], He, Li and Zhao [24]. In [39, 40, 41], Miyazawa confirmed that under an irreducible condition, there are only three types of exact tail asymptotics along a coordinate direction in a double QBD process: exact geometric, geometric multiplied by a power function with power $-1 / 2$ or $-3 / 2$.

For exact tail asymptotic analysis in the quarter plane, the theorems based on 
analysis of combinatorics is also an alternative approach. Some references include Bousquet-Melou [6] and Mishna [37].

\subsection{Contributions}

In this research, we consider a discrete-time preemptive priority model, which has not been systematically studied. Our goal is to characterize its exact tail asymptotic behaviour for the joint stationary distribution. This model has been reported in the literature, but the characterization of the tail asymptotics is not complete. As discussed previously, there are several methods of approach. In this research, we use the generating function method on the model. The main contributions made in this thesis include: (1) an explicit generating function expression and a detailed analysis of its key kernel function and zeros; (2) characterization of exact tail asymptotic behaviour along the high-priority queue direction, which is a type not following any of the three types reported in the literature for the double QBD process along a coordinate direction since the required irreducibility condition is not satisfied in our case; (3) characterization of exact tail asymptotics along the low-priority direction, our case serves as an example for the three types of tail asymptotics along a coordinate studied in Miyazawa [41]: exact geometric, a geometric multiplied by a power function with power $-1 / 2$ and $-3 / 2$; stability regions for each of the three types are provided as well.

Specifically, the following two theorems are the main contributions of this thesis.

Theorem 1.1 For the discrete-time preemptive priority queue with two classes of customers satisfying $\rho<1$, if the number of low-priority customers is fixed (i.e., a fixed number $j \geq 0$ ), the exact tail asymptotics in the joint stationary distribution 
along the high-priority queue direction is

$$
\pi_{n, j} \sim C \pi_{0,0} \frac{1}{j !} n^{j} r_{0}^{n}, \quad j \geq 0
$$

where $C, \pi_{0,0}$ and $r_{0}$ are constants given in (6.2), (3.31) and (3.43) respectively. To make the system stable, $\rho$ is defined as customer arrival probability over service time probability.

Miyazawa in [41] states there are three types of exact tail asymptoticss for the double QBD process when an irreducible condition is met. Our result is not among those three types since the irreducibility is not satisfied in our case.

Along the low-priority queue direction, there are three types of exact tail asymptotics corresponding to the three cases of the dominant singularity in the generating function $\psi_{0}(y)$ given in (3.56): a pole only; a pole and a branch point simultaneously; and a branch point only. In the probabilistic method and the matrix analytic method, these three types of tail asymptotics correspond to positive recurrent, null recurrent, and transient properties, respectively. To state the result, we partition the whole stability region into three regions according to $F\left(y_{1}\right)$, where $F\left(y_{1}\right)$ is defined in (3.64) and $y_{1}$ is the branch point of the kernel function given in (3.45).

Theorem 1.2 For the discrete-time preemptive priority queue with two classes of customers satisfying $\rho<1$, if the number of high-priority customers is fixed (i.e. a fixed number $i \geq 0$ ), the exact tail asymptotics in the joint stationary distribution along the low-priority queue direction are given below. There are three cases in total: 
(i) If $F\left(y_{1}\right)>0$, then

$$
\pi_{i, j} \sim C_{l, 1} \beta^{i}\left(\frac{1}{\eta_{1}}\right)^{j}
$$

where $\beta=\frac{\eta_{1} \bar{p} r}{p \bar{r}}$

(ii) If $F\left(y_{1}\right)=0$, then

$$
\pi_{i, j} \sim C_{l, 2} j^{-1 / 2}\left(\frac{1}{y_{1}}\right)^{j} \beta^{i}
$$

where $\beta=\sqrt{\frac{p \bar{p}}{\bar{p}}}$.

(iii) If $F\left(y_{1}\right)<0$, then

$$
\pi_{i, j} \sim C_{l, 3} j^{-3 / 2}\left(\frac{1}{y_{1}}\right)^{j}(1+H i) \beta^{i},
$$

where $\beta=\sqrt{\frac{p \bar{p}}{\bar{p} r}}$ and $H=-\frac{\psi_{0}\left(y_{1}\right) x_{1}\left(y_{1}\right)}{2 \sqrt{\pi} C_{l, 3}} \frac{q\left[(\overline{p r}-p r)^{2}\left(q y_{1}+\bar{q}\right)-(\overline{p r}+p r)\right]}{\sqrt{d\left(1-\frac{y_{1}}{y_{2}}\right)}}$

All the constants $C_{l, j}$, for $j=1,2,3$ are given in (5.2), (5.4) and (5.6), respectively.

\section{$1.3 \quad$ Organization}

This thesis is organized as follows. Chapter 2 gives a summary and review of the existing continuous-time preemptive priority queue model and lists main asympototics results of this model. Our discrete-time preemptive priority queue model description follows after that for a comparison. Expressions of generating functions and some discussions on the key kernel of the model are stated in Chapter 3. An analysis of singularities and a Tauberian-like theorem used to determine tail asymptotics provided are in Chapter 5 , which are necessary for the proofs 
of the main results in the following two chapters. Proofs and discussions of tail asymptotics along low and high priority directions are carried out in Chapter 5 and 6 , respectively. Concluding remarks and possible future work are made in the final chapter. 


\section{Chapter 2}

\section{Priority Queues -}

\section{Continuous-time or Discrete-time}

As discussed previously, the continuous-time priority queue model is well developed by multiple researchers. However, many problems we are facing in real life happen in the discrete-time situation. For example, in the telecommunications and engineering field, jobs or customers only arrive at the system around the observation instance $n$, where $n=0,1,2, \ldots$ These kinds of problems motivate the study of the discrete-time queueing model. In this thesis, we consider a discretetime preemptive priority queue. We are interested in its exact tail asymptotic behaviour in the joint stationary distribution. We will see that the continuous and discrete time preemptive priority queues share some key structural properties. For this reason, in this chapter, we first review the continuous-time preemptive priority queue model studied by Li and Zhao in [31]. Some of the main results for this continuous-time model are stated and briefly discussed here. Then, our discrete-time priority queue model is described. As we will see, the transitions for the discrete-time priority queue and the analysis of the discrete-time model are 
more challenging and more complicated than that of the continuous-time case.

\subsection{Continuous-time preemptie priority queue}

In the continuous-time preemptive priority queueing system, we consider two classes of customers and a single server. Two types of customers arrive at the system independently according to Poisson processes with rate $\lambda_{h}$ for high-priority customers and $\lambda_{l}$ for low-priority customers, respectively. For this priority queue, a high-priority customer has priority to receive service over a low-priority customer and within each type of customer, the queue follows first in first out (FIFO) discipline. For the preemptive rule, as a high-priority customer enters the system, the low-priority customer service is interrupted and will restart its service after the last high-priority customer in the system completes its service. Both classes of customers require an exponential amount of service time with different service rates $\mu_{h}$ and $\mu_{l}$, respectively. All service times are independent and also independent of the two arrival processes. Let $\lambda=\lambda_{h}+\lambda_{l}$, and without loss of generality, assume $\lambda+\mu_{h}+\mu_{l}=1$, also assume $\rho=\rho_{h}+\rho_{l}<1$, where $\rho_{h}=\frac{\lambda_{h}}{\mu_{h}}$ and $\rho_{l}=\frac{\lambda_{l}}{\mu_{l}}$, then the system is stable and we denote $\pi_{i, j}$ as the joint stationary probability distribution for the number of high- and low-priority customers respectively in the system.

The transition diagram of this continuous-time priority queue is shown in the following figure. The system equations based on the transition diagram are the 


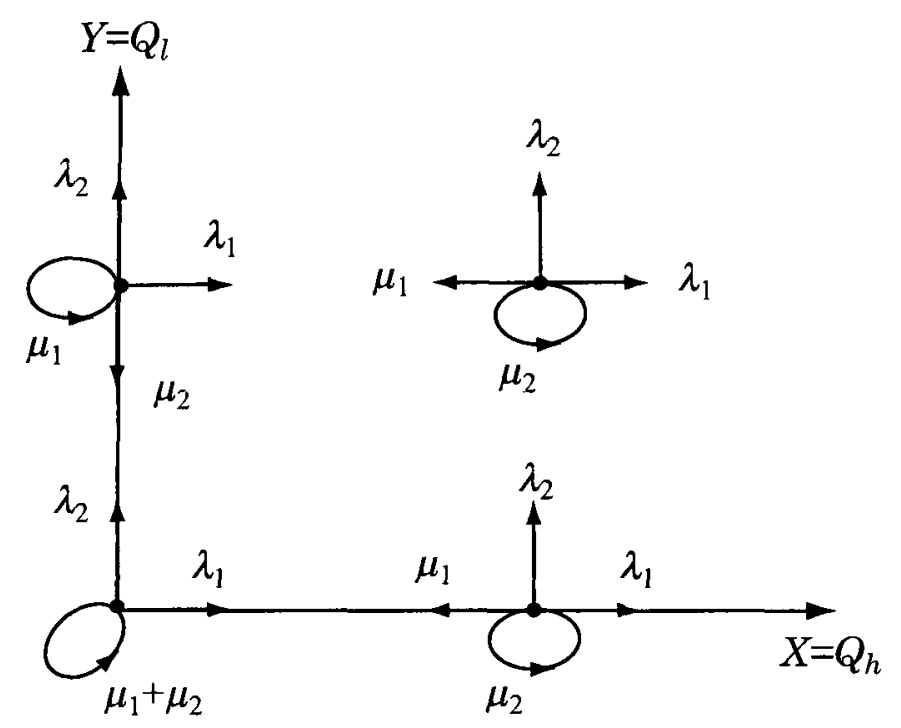

Figure 2.1: Transitions for the continuous-time priority queueing system

following:

$$
\begin{aligned}
\left(\lambda_{h}+\lambda_{l}\right) \pi_{0,0} & =\mu_{h} \pi_{1,0}+\mu_{l} \pi_{0,1}, \\
\left(\lambda_{h}+\lambda_{l}+\mu_{l}\right) \pi_{0, j} & =\mu_{h} \pi_{1, \jmath}+\mu_{l} \pi_{0, j+1}+\pi_{0, j-1}, \quad j \geq 1, \\
\left(\lambda_{h}+\lambda_{l}+\mu_{h}\right) \pi_{i, 0} & =\mu_{h} \pi_{i+1,0}+\lambda_{h} \pi_{\jmath-1,0}, \quad i \geq 1, \\
\left(\lambda_{h}+\lambda_{l}+\mu_{h}\right) \pi_{i, j} & =\mu_{h} \pi_{i+1, j}+\lambda_{h} \pi_{i-1, j}+\lambda_{l} \pi_{i, j-1}, \quad i \geq 1, \quad j \geq 1 .
\end{aligned}
$$

In [31], $\mathrm{Li}$ and Zhao use the generating function method to characterize the tail asymptotics behaviour of the continuous-time priority queue.

Proposition 2.1 (Li and Zhao [31]) For $|x| \leq 1$ and $|y| \leq 1$, the generating function of the continuous-time preemptive priority queue satisfies:

$$
\begin{aligned}
& y\left[\left(\lambda+\mu_{h}-\lambda_{l} y\right) x-\mu_{h}-\lambda_{h} x^{2}\right] P(x, y) \\
= & {\left[\left(\mu_{h}-\mu_{l}\right) x y-\mu_{h} y+\mu_{l} x\right] \psi_{0}(y)+\pi_{0,0} \mu_{l}(y-1) x, }
\end{aligned}
$$


where

$$
\pi_{0,0}=1-\rho
$$

$P(x, y)$ and $\psi_{0}(y)$ are various generating functions for the stationary probability vector $\pi_{i, j}$ given in (3.5) and (3.3) respectively.

Based on the generating functions of $\varphi_{j}(x)$ and $\psi_{0}(y)$, the exact tail asymptotics along high and low direction can be characterized. The main results for the continuous-time priority queue are stated here.

Theorem 2.1 (Li and Zhao [31]) For the classical $M / M / 1$ preemptive priority queue with two classes of customers satisfying $\rho<1$, for a fixed number $j \geq 0$ of low-priority customers, the exact tail asymptotics in the joint stationary distribution along the high-priority queue direction is characterized by:

$$
\pi_{n, j} \sim(1-\rho)\left(\frac{r_{1}^{j}}{j !}\right) n^{j} r_{0}^{n-j}
$$

where

$$
\begin{aligned}
& r_{0}=\frac{\left(\lambda+\mu_{h}\right)-\sqrt{\left(\lambda+\mu_{h}\right)^{2}-4 \lambda_{h} \mu_{h}}}{2 \mu_{h}}, \\
& r_{1}=\frac{\lambda_{l} r_{0}}{\sqrt{\left(\lambda+\mu_{h}\right)^{2}-4 \lambda_{h} \mu_{h}}} .
\end{aligned}
$$

Theorem 2.2 For the classical $M / M / 1$ preemptive priority queue with two classes of customers satisfying $\rho<1$, characterizations of the exact tail asymptotics in the joint stationary distribution along the low-priority queue direction are given below for a fixed number $i \geq 0$ of high-priority customers: (1) (Exact geometric 
decay) In the region defined by $D>0$,

$$
\pi_{\imath, n} \sim C_{l, 1}\left[\mu\left(\eta_{1}\right)\right]^{i} \eta_{1}^{n}
$$

(2) (Geometric decay with pre-factor $n^{-1 / 2}$ ) In the region defined by $D=0$,

$$
\pi_{i, n} \sim C_{l, 2}\left(\sqrt{\rho_{h}}\right)^{i} n^{-1 / 2} b_{1}^{n}
$$

(3) (Geometric decay with pre-factor $n^{-3 / 2}$ ) In the region defined by $D<0$,

$$
\pi_{i, n} \sim C_{l, 3}(1+i \widetilde{B})\left(\sqrt{\rho_{h}}\right)^{i} n^{-3 / 2} b_{1}^{n}
$$

with

$$
\begin{aligned}
D & =\left(\lambda+\mu_{h}-2 \sqrt{\lambda_{h} \mu_{h}}\right)\left(\mu_{h}-\mu_{l}-\sqrt{\lambda_{h} \mu_{h}}\right)+\lambda_{l} \mu_{l} \\
\eta_{1} & =\frac{\left(1-2 \mu_{h}\right)+\sqrt{\left(1-2 \mu_{h}\right)^{2}+4\left(\mu_{h}-\mu_{l}\right) \lambda_{l}}}{2 \mu_{l}} \\
\mu(\eta) & =\frac{1-\mu_{l}-\left(\lambda_{l} / \eta\right)-\sqrt{\left[\left(1-\mu_{l}\right)-\left(\lambda_{l} / \eta\right)\right]^{2}-4 \lambda_{h} \mu_{h}}}{2 \mu_{h}} \\
b_{1} & =\frac{\lambda_{l}}{\lambda_{l}+\left(\sqrt{\mu_{h}}-\sqrt{\lambda_{h}}\right)^{2}} \\
\widetilde{B} & =\frac{\mu_{l}-\mu_{h}-\mu_{l} b_{1}+\sqrt{\lambda_{h} \mu_{h}}}{\sqrt{\lambda_{h} \mu_{h}}} \\
C_{l, 1} & =2 a F\left(\frac{1}{\eta_{1}}\right) \\
C_{l, 2} & =a \frac{\lambda_{l} \sqrt{1-b_{2} / b_{1}}}{\sqrt{\pi} b_{1} \sqrt{b_{1} b_{2}}} \\
C_{l, 3} & =a \sigma\left(\eta_{1}\right)+b \sigma\left(\eta_{2}\right)
\end{aligned}
$$


where

$$
\begin{aligned}
F(y) & =\lambda_{l} y^{2}-\left[1-2 \mu_{h}+\mu_{l}\right] y+2 \mu_{l}, \\
\sigma(y) & =\frac{\lambda_{l} \sqrt{1-\frac{b_{2}}{b_{1}}}}{2 \sqrt{b_{1} b_{2}} \sqrt{\pi}\left(\eta-b_{1}\right)}
\end{aligned}
$$

From above we can see that there are only three types of exact tail asymptotics along the low-priority direction: exact geometric, geometric multiplied by a power function with power $-1 / 2$ or $-3 / 2$. While the result of exact tail asymptotics along the high-priority direction does not follow any of the three types since the irreducibility condition required is not satisfied here.

\subsection{Discrete-time preemptive priority queue}

In this subsection, we give a model description for the discrete-time preemptive priority queue, which is the focus of this thesis. Similarly to the continuous-time priority queue case, we also consider a system with a single server and two types of customers following the preemptive priority rule, but in discrete time. In this system, differing from the continuous-time priority queue, all possible events occur around observation instance $n$, where $n=0,1,2, \ldots$. At each time epoch, a high-priority or low-priority customer can arrive at the system independently with probability $0<p<1$ and $0<q<1$, respectively. Differing from the continuous-time preemptive priority model, a high-priority customer and a lowpriority customer can arrive at the discrete-time system simultaneously. Both types of customer require the same amount of service time, which is a geometric random variable. Equivalently, with probability $r$, a service would complete in each time slot and if there is no customer in the system, the server remains idle. 
We can view this system as a counterpart model to the classical continuous-time priority queueing system. To this system, customers arrive at the single server facility. For each of the two priority classes, one customer arrives in each time slot is a Bernoulli random variable. This means that if there is a customer entering the system, we take a successful probability and if no such event occurs during that time slot, we take a failure probability . Hence the total number of customers in the system can be described as a Binomial process. In our model, it is also clear that the inter-arrival time and the service time of the system is geometrically distributed.

For the classical Geometric/Geometric/1 model, as in our case, there are three different arrangements regarding the order in which arrivals and services take place and the time that they occur. They are early arrival system, late arrival system with immediate access and late arrival system with delayed access [26]. We consider the early arrival system here. In our model, the customer arrival is observed at epoches $n$; at completion of the service, the customer leaves the system at $n-$ before the observation epoch; and the new customer arrival occurs at the early $n+$ of the next time interval, who enters the service facility immediately, if the system is free. This process is shown in Fig 2.2. Similar to the continuous-time

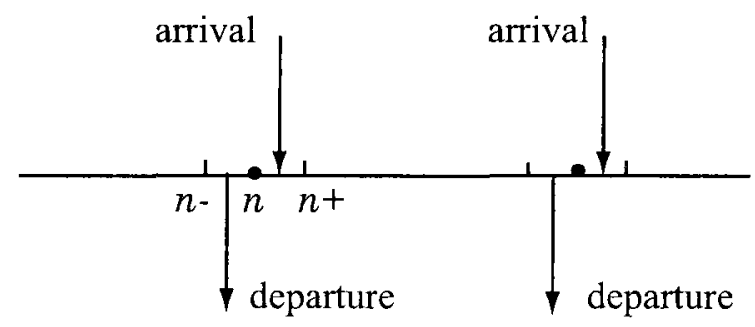

Figure 2.2: Time instances of arrivals, departures and observations

priority case, we assume that a high-priority customer has priority over any low- 
priority customer to receive the service. In each of these two classes, the customers are served according to the FIFO queueing discipline. With the preemptive rule, the service of the low-priority customer is interrupted upon the arrival of a highpriority customer. The interrupted low-priority customer will stay at the head of the line waiting for restart of the service immediately after the last high-priority customer in the system completes its service.

Let $Q_{1}(n)$ and $Q_{2}(n)$ be the number of low-priority and high-priority customers in the system, including the customer that is in the service, respectively. It is clear that $Q_{1}(n), Q_{2}(n) ; n=0,1,2, \ldots$ is a discrete-time Markov chain. We let $\lambda=p+q$ and assume that $\rho=\rho_{h}+\rho_{l}<1$ for the stability of the system, where $\rho_{h}=\frac{p}{r}<1$ and $\rho_{l}=\frac{q}{r}<1$. Under this condition, we denote $\pi_{i, j}$ the joint stationary probability distribution for the number of low- and high-priority customers, respectively, in the system. We also use the following convention: for two functions $f(n)$ and $g(n)$ of nonnegative integers $n, f(n) \sim g(n)$ means that $\lim _{n \rightarrow \infty} \frac{f(n)}{g(n)}=1$. Our goal is to characterize tail asymptotics for the stationary distribution $\pi_{i, j}$, or to find a function $g(n)$ such that for each $j$, we have $\lim _{n \rightarrow \infty} \frac{\pi_{n, j}}{g(n)}=c_{j}$, where $g(n)$ is independent of $j$; or symmetrically, to find a function $g(n)$ such that for each $i$, we have $\lim _{n \rightarrow \infty} \frac{\pi_{\imath, n}}{g(n)}=c_{i}$, where $g(n)$ is independent of $i$.

The transition diagram of this Markov chain is shown on the next page, and based on $p_{0, j}$ and $p_{0, j}^{(h)}, h=0,1,2$, transition probability can be easily determined. Since a high-priority customer has priority over any low-priority customer, transitions can only occur to an upward direction except for the case that there is no highpriority customer or $p_{0,-1}^{(2)}$ can be positive. Then obviously for this reason, the one-dimensional process or random walk defined by $p_{j}=\sum_{\imath} p_{i, j}$ with the reflected boundary defined by $p_{j}^{(1)}=\sum_{i} p_{i, j}^{(1)}$ for $j=0,1$ is not irreducible. Comparing with the continuous-time priority queue model, we can also see that the discrete- 
time priority queue is more complicated. In the discrete-time case, we also have transition along a triangular direction with a positive probability. The balance

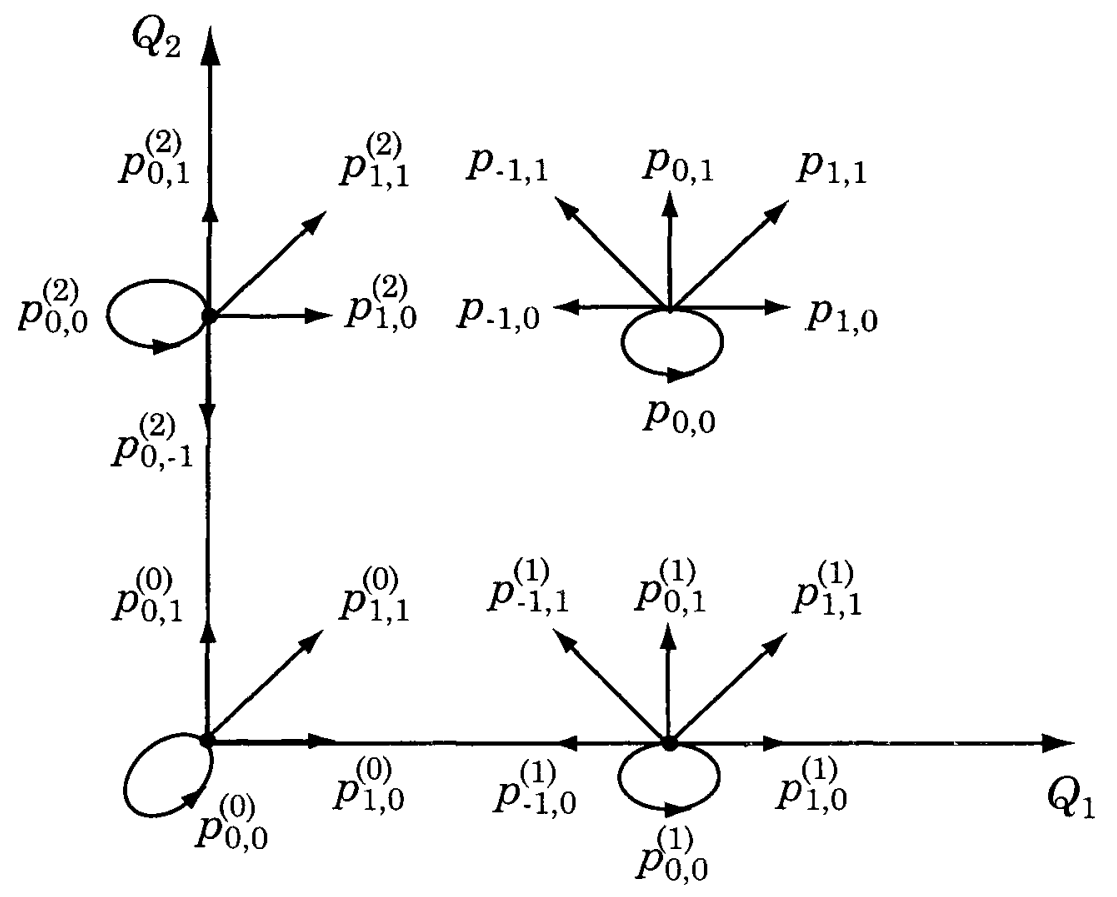

Figure 2.3: Transitions for the discrete-time priority queueing system

equations of the system for the discrete-time preemptive priority queueing model are the following based on the transition diagram in Fig 2.3:

$$
\begin{array}{r}
\left(p_{0,1}^{(0)}+p_{1,1}^{(0)}+p_{1,0}^{(0)}\right) \pi_{0,0}=p_{0,-1}^{(2)} \pi_{0,1}+p_{-1,0}^{(1)} \pi_{1,0}, \\
\left(p_{0,1}^{(2)}+p_{1,1}^{(2)}+p_{1,0}^{(2)}+p_{0,-1}^{(2)}\right) \pi_{0, j}=p_{0,1}^{(0)} \pi_{0, j-1}+p_{-1,0} \pi_{1, j} \\
+p_{-1,1} \pi_{1, j-1}+p_{0,-1} \pi_{0, j+1}, \quad j \geq 1, \\
\left(p_{-1,0}^{(1)}+p_{-1,1}^{(1)}+p_{0,1}^{(1)}+p_{1,1}^{(1)}+p_{1,0}^{(1)}\right) \pi_{i, 0}=p_{1,0}^{(0)} \pi_{i-1,0}+p_{-1,0}^{(1)} \pi_{\imath+1,0}, \\
i \geq 1, \\
\left(p_{-1,0}+p_{-1,1}+p_{0,1}+p_{1,1}+p_{1,0}\right) \pi_{i, j}=p_{1,0} \pi_{l-1, j}+p_{-1,0} \pi_{\imath+1, j} \\
+p_{0,1} \pi_{i, j-1}+p_{1,1} \pi_{i-1, j-1}+p_{-1,1} \pi_{\imath+1, j-1}, \quad i \geq 1, \quad j \geq 1 .
\end{array}
$$


Our goal is to characterize the exact tail asymptotics for the joint stationary distribution $\pi_{i, j}$ of this discrete-time preemptive priority queue described above. In the following chapters, we use the generating function method for this purpose. The balance equations from (2.23) to (2.26) are used to derive the generating function of the system. 


\section{Chapter 3}

\section{Generating Functions and the Kernel}

There are several approaches to characterize the exact tail asymptotics of the joint stationary distribution for the discrete-time priority queue model. For our case, we use the generating function method. In this chapter, first we determine the fundamental form of our model based on a modification of the result provided by Fayolle, Iasnogorodski and Malyshev [10]. Then. we do some analysis on the key kernel equation. Finally we characterize the generating functions $\varphi_{0}(x)$ and $\psi_{0}(y)$, which are the key for the analysis of exact tail asymptotics along the high and low priority directions in the later chapters.

\subsection{Fundamental form}

Our discrete-time priority queueing system is a specific case of random walks in the quarter plane studied in Fayolle, Iasnogorodski and Malyshev [10]. We 
define various generating functions for the stationary probability vector $\pi_{i, j}$ as the following:

$$
\begin{aligned}
\varphi_{0}(x) & =\sum_{i=0}^{\infty} \pi_{i, 0} x^{i} \\
\varphi_{j}(x) & =\sum_{i=0}^{\infty} \pi_{i, j} x^{i}, \quad j \geq 1 \\
\psi_{0}(y) & =\sum_{j=0}^{\infty} \pi_{0, j} y^{j}, \\
\psi_{i}(y) & =\sum_{j=0}^{\infty} \pi_{i, j} y^{j}, \quad i \geq 1 \\
P(x, y) & =\sum_{j=0}^{\infty} \varphi_{j}(x) y^{j}=\sum_{i=0}^{\infty} \psi_{i}(y) x^{i} .
\end{aligned}
$$

We know that $\varphi_{0}(x)=P(x, 0)=P_{1}(x)$ and $\psi_{0}(y)=P(0, y)=P_{2}(y)$.

Fayolle, Iasnogorodski and Malyshev [10] provided a method to obtain the socalled fundamental form for the generating function of joint probabilities $\pi_{i, j}$ for $i \geq 1$ (excluding $i=0$ or $j=0$ ). We use the same idea to obtain the fundamental form for $P(x, y)$ of $\pi_{i, j}$ for all values of $i, j \geq 0$. First, we define the followings:

$$
\begin{aligned}
h(x, y) & =x y\left(1-\sum_{i=-1}^{1} \sum_{j=-1}^{1} p_{i, j} x^{i} y^{j}\right) \\
& =\widetilde{a}(x) y^{2}+\widetilde{b}(x) y+\widetilde{c}(x), \\
h_{1}(x, y) & =x\left(\sum_{i=-1}^{1} \sum_{j=0}^{1} p_{i, j}^{(1)} x^{i} y^{j}-1\right) \\
& =\left(p_{-1,0}^{(1)}+p_{0,0}^{(1)} x+p_{1,0}^{(1)} x^{2}\right)+\left(p_{-1,1}^{(1)}+p_{0,1}^{(1)} x+p_{1,1}^{(1)} x^{2}\right) y-x, \\
h_{2}(x, y) & =y\left(\sum_{i=0}^{1} \sum_{j=-1}^{1} p_{i, j}^{(2)} x^{i} y^{j}-1\right) \\
& =\left(p_{0,-1}^{(2)}+p_{0,0}^{(2)} y+p_{0,1}^{(2)} y^{2}\right)+\left(p_{1,-1}^{(2)}+p_{1,0}^{(2)} y+p_{1,1}^{(2)} y^{2}\right) x-y,
\end{aligned}
$$




$$
\begin{aligned}
h_{0}(x, y) & =\left(\sum_{i=0}^{1} \sum_{j=0}^{1} p_{i, j}^{(0)} x^{i} y^{j}-1\right) \\
& =\left(p_{0,0}^{(0)}+p_{1,0}^{(0)} x+p_{0,1}^{(0)} y+p_{1,1}^{(0)} x y\right)-1,
\end{aligned}
$$

where

$$
\begin{aligned}
& \widetilde{a}(x)=-\left(p_{-1,1}+p_{0,1} x+p_{1,1} x^{2}\right), \\
& \widetilde{b}(x)=-\left[p_{-1,0}+\left(p_{0,0}-1\right) x+p_{1,0} x^{2}\right], \\
& \widetilde{c}(x)=-\left(p_{-1,-1}+p_{0,-1} x+p_{1,-1} x^{2}\right) .
\end{aligned}
$$

Lemma 3.1 The generating function $P(x, y)$ takes the following fundamental for$m:$

$$
K(x, y) P(x, y)=H_{1}(x, y) P_{1}(x)+H_{2}(x, y) P_{2}(y)+H_{0}(x, y) \pi_{0,0}
$$

where

$$
\begin{aligned}
K(x, y) & =h(x, y) \\
H_{1}(x, y) & =h(x, y)+h_{1}(x, y) y \\
H_{2}(x, y) & =h(x, y)+h_{2}(x, y) x \\
H_{0}(x, y) & =h_{0}(x, y) x y-h(x, y)-h_{1}(x, y) y-h_{2}(x, y) x .
\end{aligned}
$$

It is clear to see that in order to characterize the generating function, we first need to define the transition probabilities in our discrete-time priority queuing model. Based on the transition diagram in Fig 2.3, the transition probabilities are given 
below:

$$
\begin{aligned}
p_{1,0}^{(0)} & =p_{1,0}^{(1)}=p_{1,0}^{(2)}=p_{1,0}=p \overline{q r} \\
p_{1,1}^{(0)} & =p_{1,1}^{(1)}=p_{1,1}^{(2)}=p_{1,1}=p q \bar{r} \\
p_{0,1}^{(0)} & =p_{0,1}^{(1)}=p_{0,1}^{(2)}=p_{0,1}=q(\overline{p r}+p r) \\
p_{-1,1} & =\bar{p} q r \\
p_{-1,0}^{(1)} & =p_{-1,0}=\overline{p q} r \\
p_{0,0}^{(1)} & =p_{0,0}=\overline{p q r}+p \bar{q} r \\
p_{0,0}^{(2)} & =\overline{p q r}+p \bar{q} r+\bar{p} q r \\
p_{0,0}^{(0)} & =\overline{p q}+p \bar{q} r+\bar{p} q r \\
p_{0,-1}^{(2)} & =\overline{p q} r
\end{aligned}
$$

where

$$
\bar{p}=1-p, \quad \bar{q}=1-q, \quad \bar{r}=1-r .
$$

These transition probabilities are derived from the transition diagram. Taking equation (3.20) as an example, there are only two possible cases that can lead to the result of $p_{0,1}$. One case is that if there is no high-priority customer coming and meanwhile the low-priority customer is not being served, we then have the transition probability equal to $\bar{p} q \bar{r}$. The other case is if there is that one highpriority customer coming and it is served immediately, and meanwhile nothing happens to the low-priority customer, we then have the transition probability equal to pqr. Combining these two cases leads to the transition probability of $p_{0,1}$. The same idea is used to derive all the rest of the transition probabilities.

Now, we use these determinations of the transition probabilities and equations 
(3.6) to find $h(x, y)$ :

$$
\begin{aligned}
h(x, y) & =x y\left(1-\sum_{i=-1}^{1} \sum_{j=-1}^{1} p_{i, j} x^{i} y^{j}\right) \\
& =x y\left[1-\left(p_{0,0}+p_{1,0} x+p_{1,1} x y+p_{0,1} y+p_{-1,1} x^{-1} y+p_{-1,0} x^{-1}\right)\right] \\
& =\widetilde{a}(x) y^{2}+\widetilde{b}(x) y+\widetilde{c}(x),
\end{aligned}
$$

where

$$
\begin{aligned}
& \tilde{a}(x)=-\left(p_{-1,1}+p_{0,1} x+p_{1,1} x^{2}\right)=-\bar{p} q r-(\bar{p} q \bar{r}+p q r) x-p q \bar{r} x^{2}, \\
& \widetilde{b}(x)=-\left[p_{-1,0}+\left(p_{0,0}-1\right) x+p_{1,0} x^{2}\right]=-\overline{p q} r-(\overline{p q r}+p \bar{q} r-1) x-p \overline{q r} x^{2} \\
& \widetilde{c}(x)=-\left(p_{-1,-1}+p_{0,-1} x+p_{1,-1} x^{2}\right)=0
\end{aligned}
$$

Hence, from equation (3.14) we have,

$$
K(x, y)=-\left[\bar{p} q r+(\bar{p} q \bar{r}+p q r) x+p q \bar{r} x^{2}\right] y^{2}-\left[\overline{p q} r+(\overline{p q r}+p \bar{q} r-1) x+p \bar{q} r x^{2}\right] y
$$

To make work easier later, we derive the following:

$$
\begin{aligned}
y h_{1}(x, y) & =x y\left(p_{1,0} x+p_{1,1} x y+p_{0,1} y+p_{-1,1} x^{-1} y+p_{-1,0} x^{-1}+p_{0,0}^{(1)}-1\right), \\
x h_{2}(x, y) & =x y\left(p_{0,-1}^{(2)} y^{-1}+p_{0,0}^{(2)}+p_{0,1} y+p_{1,1} x y+p_{1,0} x-1\right), \\
x y h_{0}(x, y) & =x y\left(p_{1,0} x+p_{1,1} x y+p_{0,1} y+p_{0,0}^{(0)}-1\right) .
\end{aligned}
$$


Now, we use equation (3.15), (3.16) and (3.17) to derive $H_{1}(x, y), H_{2}(x, y)$ and $H_{0}(x, y)$, respectively.

$$
\begin{aligned}
& H_{1}(x, y) \\
& =x y\left[1-\left(p_{0,0}+p_{1,0} x+p_{1,1} x y+p_{0,1} y+p_{-1,1} x^{-1} y+p_{-1,0} x^{-1}\right)\right] \\
& +x y\left(p_{1,0} x+p_{1,1} x y+p_{0,1} y+p_{-1,1} x^{-1} y+p_{-1,0} x^{-1}+p_{0,0}^{(1)}-1\right) \\
& =0 \\
& H_{2}(x, y) \\
& =x y\left[p_{0,-1}^{(2)} y^{-1}+p_{0,0}^{(2)}-p_{0,0}-p_{-1,1} x^{-1} y-p_{-1,0} x^{-1}\right] \\
& =x y\left[\overline{p q} r y^{-1}+(\bar{p} \bar{q}+p \bar{q} r+\bar{p} q r)-(\overline{p q r}+p \bar{q} r)-\bar{p} q r x^{-1} y-\overline{p q} r x^{-1}\right] \\
& =\overline{p q} r x+\bar{p} q r x y-\bar{p} q r y^{2}-\overline{p q} r y \text {, } \\
& H_{0}(x, y) \\
& =h_{0}(x, y) x y-h(x, y)-h_{1}(x, y) y-h_{2}(x, y) x \\
& =h_{0}(x, y) x y-h_{2}(x, y) x \\
& =x y\left(p_{1,0} x+p_{1,1} x y+p_{0,1} y+p_{0,0}^{(0)}-1\right)-x y\left(p_{0,-1}^{(2)} y^{-1}+p_{0,0}^{(2)}+p_{0,1} y+p_{1,1} x y+p_{1,0} x-1\right) \\
& =x y\left(p_{0,0}^{(0)}-p_{0,-1}^{(2)} y^{-1}-p_{0,0}^{(2)}\right) \\
& =x y\left(\overline{p q}-\overline{p q} r y^{-1}-\overline{p q r}\right) \\
& =\overline{p q} r x(y-1) \text {. }
\end{aligned}
$$

As we can see, to characterize the generating function besides $\psi_{0}(y)$, we still need to specify $\pi_{0,0}$.

\section{Proposition 3.1}

$$
\pi_{0,0}=1-\frac{(p+q) \bar{r}+p q r}{\overline{p q} r}
$$


Proof. From the previous calculations, we know $H_{1}(x, y)=0$. This gives $K(x, y) P(x, y)=H_{2}(x, y) P_{2}(y)+H_{0}(x, y) \pi_{0,0}$. Further we set $H_{2}(x, y)=0$, a connection between $x$ and $y$ can be derived from the following steps.

$$
\begin{aligned}
H_{2}(x, y) & =\overline{p q} r x+\bar{p} q r x y-\bar{p} q r y^{2}-\overline{p q} r y=0, \\
\bar{q} x+q x y-q y^{2}-\bar{q} y & =0, \\
x(\bar{q}+q y) & =y(q y+\bar{q}), \\
x & =y, \quad \text { if } q y+\bar{q} \neq 0 .
\end{aligned}
$$

Hence we have the following:

$$
\begin{aligned}
K(x, y) P(x, y) & =H_{0}(x, y) \pi_{0,0} \\
\pi_{0,0} & =\lim _{x \rightarrow 1} \frac{K(x, y(x)) P(x, y(x))}{H_{0}(x, y(x))} .
\end{aligned}
$$

Then using the relationship we found between $x$ and $y$ by setting $H_{2}(x, y)=0$, we have

$$
=\lim _{x \rightarrow 1}^{\pi_{0,0}} \frac{\left\{\bar{p} q r x^{2}+\bar{p} q \bar{r} x^{3}+p q r x^{3}+p q \bar{r} x^{4}+\overline{p q} r x+\overline{p q r} x^{2}+p \bar{q} r x^{2}-x^{2}+p \overline{q r} x^{3}\right\} P(x, x)}{-\bar{p} \bar{q} r x(x-1)}
$$

Substitute $x=1$, we find that the above limit is a typical $\frac{0}{0}$ type in calculus. By L'Hospital's rule, taking the derivatives of both numerator and denominator, we 
can find $\pi_{0,0}$ :

$$
\begin{aligned}
& \pi_{0,0} \\
= & \lim _{x \rightarrow 1} \frac{\left\{2 x \bar{p} q r+3 x^{2}(\bar{p} q \bar{r}+p q r)+4 x^{3} p q \bar{r}+\overline{p q} r+2 x \overline{p q r}+2 x p \bar{q} r-2 x+3 x^{2} p \bar{q} \bar{r}\right\} P(1,1)}{-\overline{p q} r(2 x-1)} \\
= & \frac{2 \bar{p} r q+3 q \overline{p r}+3 p q r+4 p q \bar{r}+\overline{p q} r+2 \overline{p q r}+2 p \bar{q} r-2+3 p \overline{q r}}{-\overline{p q} r} \\
= & \frac{r q(2+p)+q \bar{r}(3+p)+p \bar{q}(3-r)+\overline{p q}(2-r)}{-\overline{p q} r} \\
= & \frac{q(2+p)+q \bar{r}+\bar{q}(2-r)+p \bar{q}-2}{-\overline{p q} r} \\
= & \frac{r-(p+q)}{\overline{p q} r} \\
= & 1-\frac{(p+q) \bar{r}+p q r}{\overline{p q} r}
\end{aligned}
$$

The proof is completed.

From the expression of $\pi_{0,0}$, if there is only one type of customer in the model, i.e. we set $q=0$, we will have $\pi_{0,0}=1-\frac{p \bar{r}}{\bar{p} r}$. This is exactly the same result of $\pi_{0,0}$ for the well known $M / M / 1$ queue.

Now we are ready to write the generating function for our model.

Lemma 3.2 For $|x| \leq 1$ and $|y| \leq 1$, we have the following expression for the generating function for the discrete-time preemptive priority queueing system:

$$
P(x, y)=\frac{H_{2}(x, y) \psi_{0}(y)+H_{0}(x, y) \pi_{0,0}}{K(x, y)}
$$

where $H_{2}(x, y) H_{0}(x, y) K(x, y)$ and $\pi_{0,0}$ are given in (3.29) (3.30) (3.27) and (3.31), respectively.

Proof. This can be easily done by substituting our $H_{1}(x, y) H_{2}(x, y) H_{0}(x, y)$ $H(x, y)$ and $\pi_{0,0}$ to fundamental form in $(3.13)$ and rewrite $P(x, y)$. 


\subsection{Key kernel}

In the fundamental form $(3.13), K(x, y)$ is called the kernel of the generating function. For our discrete-time priority queue, $H_{1}(x, y)=0$, and the kernel $K(x, y)$ is not irreducible (as a polynomial of $x$ and $y$ ). We define $K(x, y)=-y K_{0}(x, y)$, where $K_{0}(x, y)$ is called the key kernel of the generating function. We have,

$$
\begin{aligned}
K_{0}(x, y) & =\frac{K(x, y)}{-y} \\
& =\left(p_{-1,1}+p_{0,1} x+p_{1,1} x^{2}\right) y+p_{-1,0}+\left(p_{0,0}-1\right) x+p_{1,0} x^{2} \\
& =\left(p_{1,1} y+p_{1,0}\right) x^{2}+\left(p_{0,1} y+p_{0,0}-1\right) x+\left(p_{-1,1} y+p_{-1,0}\right) \\
& =a(y) x^{2}+b(y) x+c(y)
\end{aligned}
$$

where

$$
\begin{aligned}
& a(y)=p_{1,1} y+p_{1,0}=p \bar{r}(q y+\bar{q}) \\
& b(y)=p_{0,1} y+p_{0,0}-1=(\overline{p r}+p r)(q y+\bar{q})-1, \\
& c(y)=p_{-1,1} y+p_{-1,0}=\bar{p} r(q y+\bar{q}) .
\end{aligned}
$$

Zeros of the key kernel $K_{0}(x, y)$ play an important role in the analysis of asymptotics. In the following, we provide a basic property of the zeros. For each fixed $y$, we consider $K_{0}(x, y)$ as a polynomial of $x$. The two zeros are given by

$$
\begin{aligned}
x_{1}(y) & =\frac{-\left(p_{0,1} y+p_{0,0}-1\right)+\sqrt{\Delta(y)}}{2\left(p_{1,1} y+p_{1,0}\right)} \\
& =\frac{-b(y)+\sqrt{\Delta(y)}}{2 a(y)}
\end{aligned}
$$




$$
\begin{aligned}
x_{2}(y) & =\frac{-\left(p_{0,1} y+p_{0,0}-1\right)-\sqrt{\Delta(y)}}{2\left(p_{1,1} y+p_{1,0}\right)} \\
& =\frac{-b(y)-\sqrt{\Delta(y)}}{2 a(y)}
\end{aligned}
$$

where

$$
\begin{aligned}
\Delta(y) & =\left(p_{0,1} y+p_{0,0}-1\right)^{2}-4\left(p_{1,1} y+p_{1,0}\right)\left(p_{-1,1} y+p_{-1,0}\right) \\
& =b^{2}(y)-4 a(y) c(y) .
\end{aligned}
$$

For the two zeros, when $y=0$, we have

$$
\begin{aligned}
& x_{1}(0)=\frac{-\left(p_{0,0}-1\right)+\sqrt{\left(p_{0,0}-1\right)^{2}-4 p_{1,0} p_{-1,0}}}{2 p_{1,0}}, \\
& x_{2}(0)=\frac{-\left(p_{0,0}-1\right)-\sqrt{\left(p_{0,0}-1\right)^{2}-4 p_{1,0} p_{-1,0}}}{2 p_{1,0}} .
\end{aligned}
$$

These can be simplified to the following:

$$
\begin{aligned}
& x_{1}(0)=\frac{1}{r_{0}}, \\
& x_{2}(0)=\frac{r_{0}}{w},
\end{aligned}
$$

where,

$$
\begin{aligned}
r_{0} & =\frac{1}{x_{1}(0)}, \\
w & =\left[\frac{2 p_{1,0}}{-\left(p_{0,0}-1\right)-\sqrt{\left(p_{0,0}-1\right)^{2}-4 p_{1,0} p_{-1,0}}}\right]\left[\frac{2 p_{1,0}}{-\left(p_{0,0}-1\right)+\sqrt{\left(p_{0,0}-1\right)^{2}-4 p_{1,0} p_{-1,0}}}\right] \\
& =\frac{p \overline{q r}}{\overline{p q} r}=\left(\frac{p \bar{q}}{\bar{p} q}\right) /\left(\frac{r}{\bar{r}}\right) .
\end{aligned}
$$

In the following, we determine the branch points of the kernel function, which are defined as the zeros of $\Delta(y)$. To make things easier, we let $Y=q y+\bar{q}$, then $\Delta(y)$ 
can be simplified to the following,

$$
\begin{aligned}
\Delta(y) & =b^{2}(y)-4 a(y) c(y) \\
& =[(\overline{p r}+p r) Y-1]^{2}-4 p \overline{r p} r Y^{2} \\
& =\left[(\overline{p r}+p r)^{2}-4 p \overline{r p} r\right] Y^{2}-2(\overline{p r}+p r) Y+1 \\
& =(\overline{p r}-p r)^{2} Y^{2}-2(\overline{p r}+p r) Y+1
\end{aligned}
$$

So solving $\Delta(y)=0$ gives

$$
\begin{aligned}
Y & =\frac{2(\overline{p r}+p r) \pm \sqrt{4(\overline{p r}+p r)^{2}-4(\overline{p r}-p r)^{2}}}{2(\overline{p r}-p r)^{2}} \\
& =\frac{(\overline{p r}+p r) \pm \sqrt{(\overline{p r}+p r)^{2}-(\overline{p r}-p r)^{2}}}{(\overline{p r}-p r)^{2}} \\
& =\frac{(\overline{p r}+p r) \pm 2 \sqrt{\overline{p r} p r}}{(\overline{p r}-p r)^{2}} .
\end{aligned}
$$

Hence, the branch points of the kernel are

$$
\begin{aligned}
y_{1} & =\frac{(\overline{p r}+p r)-2 \sqrt{\overline{p r} p r}}{(\overline{p r}-p r)^{2} q}-\frac{\bar{q}}{q} \\
y_{2} & =\frac{(\overline{p r}+p r)+2 \sqrt{\overline{p r} p r}}{(\overline{p r}-p r)^{2} q}-\frac{\bar{q}}{q} .
\end{aligned}
$$

Lemma 3.3 Let $y_{b}$ be the zero of $b(y)$, then we have $y_{2}>y_{b}>y_{1}>1$.

Proof. When $y<1$, we have $q y+\bar{q}<1$, combining with $\overline{p r}+p r<1$, we have $b(y)=(\overline{p r}+p r)(q y+\bar{q})-1<0$. This indicates that we must have $y_{b}>1$ since $b(y)$ is a linear function. We also know $a(1)+b(1)+c(1)=p \bar{r}+\overline{p r}+p r-1+\bar{p} r=0$. 
Hence we have,

$$
\begin{aligned}
\Delta(1) & =b^{2}(1)-4 a(1) c(1) \\
& =(a(1)+c(1))^{2}-4 a(1) c(1) \\
& =(a(1)-c(1))^{2}>0 .
\end{aligned}
$$

Also, we have $\Delta\left(y_{b}\right)=-4 a\left(y_{b}\right) c\left(y_{b}\right)<0$, then by the Mean Value Theorem from calculus, we know $\Delta(y)$ has a root existing in the interval $\left(1, y_{b}\right)$. Similarly, since $\Delta(y) \rightarrow \infty$ as $y \rightarrow \infty$, then $\Delta(y)$ also has a root existing in the interval $\left(y_{b}, \infty\right)$. It is obviously that $y_{2}>y_{1}$, hence we have $y_{2}>y_{b}>y_{1}>1$, which completes the proof.

This result indicates that $y_{1}$ is the smaller non-negative branch point of the kernel function.

Lemma 3.4 For $-1 \leq y \leq 1$, we have $x_{2}(y)<x_{1}(y)$. When $y=0$, let $x_{1}=x_{1}(0)$ and $x_{2}=x_{2}(0)$, then we have $x_{2}<1<x_{1}$.

Proof. It is clear that $x_{2}(y)<x_{1}(y)$. To show $x_{2} \leq 1$, we just need to show

$$
\frac{-\left(p_{0,0}-1\right)-\sqrt{\left(p_{0,0}-1\right)^{2}-4 p_{1,0} p_{-1,0}}}{2 p_{1,0}}<1 .
$$

With $2 p_{1,0}>0$, the above inequality can be simplified to the following

$$
\begin{aligned}
-\left(p_{0,0}-1\right)-\sqrt{\left(p_{0,0}-1\right)^{2}-4 p_{1,0} p_{-1,0}} & <2 p_{1,0} \\
-\sqrt{\left(p_{0,0}-1\right)^{2}-4 p_{1,0} p_{-1,0}} & <2 p_{1,0}+p_{0,0}-1 \\
\left(p_{0,0}-1\right)^{2}-4 p_{1,0} p_{-1,0} & >4 p_{1,0}^{2}+\left(p_{0,0}-1\right)^{2}+4 p_{1,0}\left(p_{0,0}-1\right)
\end{aligned}
$$




$$
\begin{aligned}
-p_{-1,0} & >p_{1,0}+p_{0,0}-1 \\
-\overline{p q} r & >p \overline{q r}+\overline{p q r}+p \bar{q} r-1 \\
-\overline{p q} r & >p \bar{q}+\overline{p q r}-1 \\
1 & >p \bar{q}+\overline{p q r}+\overline{p q} r \\
1 & >p \bar{q}+\overline{p q} \\
1 & >\bar{q} \\
q & >0,
\end{aligned}
$$

which is obviously true. Hence we have shown that the smaller zero $x_{2}(y)$ is inside the unit circle when $-1 \leq y \leq 1$.

\section{Corollary 3.1}

$$
\Delta(y)=d\left(1-\frac{y}{y_{1}}\right)\left(1-\frac{y}{y_{2}}\right)
$$

where

$$
d=1-(\overline{p r}+p r)+\bar{q}^{2},
$$

$y_{1}$ and $y_{2}$ are the branch points given by (3.45), (3.46) respectively.

Proof. Simple algebra is used to derive $d$.

$$
\begin{aligned}
\Delta(y) & =\frac{d}{y_{1} y_{2}}\left(y_{1}-y\right)\left(y_{2}-y\right) \\
& =(\overline{p r}-p r)^{2} Y^{2}-2(\overline{p r}+p r) Y+1 . \\
\frac{d}{y_{1} y_{2}} & =[(\overline{p r}-p r) q]^{2} .
\end{aligned}
$$




$$
\begin{aligned}
d & =y_{1} y_{2}[(\overline{p r}-p r) q]^{2} \\
& =\frac{\left(Y_{1}-\bar{q}\right)\left(Y_{2}-\bar{q}\right)}{q^{2}}[(\overline{p r}-p r) q]^{2} \\
& =\left[Y_{1} Y_{2}-\left(Y_{1}+Y_{2}\right) \bar{q}+\bar{q}^{2}\right](\overline{p r}-p r)^{2} \\
& =\left\{\frac{(\overline{p r}+p r)^{2}-4 \overline{p r} p r}{(\overline{p r}-p r)^{4}}-\frac{2(\overline{p r}+p r)}{(\overline{p r}-p r)^{2}} \bar{q}+\bar{q}^{2}\right\}(\overline{p r}-p r)^{2} \\
& =\frac{(\overline{p r}+p r)^{2}-4 \overline{p r} p r-(\overline{p r}+p r)(\overline{p r}-p r)^{2}+\bar{q}^{2}(\overline{p r}-p r)^{2}}{(\overline{p r}-p r)^{2}} \\
& =1-(\overline{p r}+p r)+\bar{q}^{2} .
\end{aligned}
$$

This simplified notation of $\Delta(y)$ is useful later for determining the exact tail asymptotics of the joint stationary distribution.

\subsection{Generating functions $\varphi_{j}(x)$ and $\psi_{0}(y)$}

In order to find the explicit expression for the generating function $P(x, y)$, we need to determine $\psi_{0}(y)$. The general approach is to consider zeros of the kernel defined by $K(x, y)=0$. Note that in our model we have $P_{1}(x)=P(x, 0)=\varphi_{0}(x)$ and $H_{1}(x, y)=0$. For this priority queue, the kernel $K(x, y)=0$ is not irreducible (as a polynomial of $x$ and $y$ ). We find it more convenient to characterize $\varphi_{\jmath}(y)$, which is used for tail asymptotics along the high-priority queue direction, directly based on the system balance equations.

Using balance equation (2.25), we have

$$
\begin{aligned}
& \left(p_{-1,0}+p_{-1,1}+p_{0,1}+p_{1,1}+p_{1,0}\right) \varphi_{0}(x)-\left(p_{-1,0}+p_{-1,1}+p_{0,1}+p_{1,1}+p_{1,0}\right) \pi_{0,0} \\
= & p_{1,0} x \varphi_{0}(x)+p_{-1,0} \frac{1}{x} \varphi_{0}(x)-p_{-1,0} \frac{1}{x}\left(\pi_{0,0}+x \pi_{1,0}\right),
\end{aligned}
$$




$$
\begin{aligned}
\varphi_{0}(x) & =\frac{\left(p_{-1,0}+p_{-1,1}+p_{0,1}+p_{1,1}+p_{1,0}\right) \pi_{0,0}-p_{-1,0} \frac{1}{x}\left(\pi_{0,0}+x \pi_{1,0}\right)}{p_{-1,0}+p_{-1,1}+p_{0,1}+p_{1,1}+p_{1,0}-p_{1,0} x-p_{-1,0} \frac{1}{x}} \\
& =\frac{p_{-1,0}\left(\pi_{0,0}+x \pi_{1,0}\right)-x \pi_{0,0}\left(p_{-1,0}+p_{-1,1}+p_{0,1}+p_{1,1}+p_{1,0}\right)}{p_{1,0} x^{2}-\left(p_{-1,0}+p_{-1,1}+p_{0,1}+p_{1,1}+p_{1,0}\right) x+p_{-1,0}} \\
& =\frac{p_{-1,0}\left(\pi_{0,0}+x \pi_{1,0}\right)-x \pi_{0,0}\left(p_{-1,0}+p_{-1,1}+p_{0,1}+p_{1,1}+p_{1,0}\right)}{p_{1,0}\left(x-x_{1}\right)\left(x-x_{2}\right)}
\end{aligned}
$$

where $x_{1}$ and $x_{2}$ are shown in equations (3.39) and (3.40) respectively.

Similarly, using balance equation (2.26), we can obtain $\varphi_{j}(x)$ as the following,

$$
\begin{gathered}
\left(p_{-1,0}+p_{-1,1}+p_{0,1}+p_{1,1}+p_{1,0}\right) \varphi_{j}(x)-\left(p_{-1,0}+p_{-1,1}+p_{0,1}+p_{1,1}+p_{1,0}\right) \pi_{0, j} \\
=p_{1,0} x \varphi_{j}(x)+\left[p_{-1,0} \frac{1}{x} \varphi_{j}(x)-p_{-1,0} \frac{1}{x}\left(\pi_{0, j}+x \pi_{1, j}\right)\right]+\left[p_{0,1} \varphi_{j-1}(x)-p_{0,1} \pi_{0, j-1}\right] \\
+p_{1,1} x \varphi_{j-1}(x)+\left[p_{-1,1} \frac{1}{x} \varphi_{j-1}(x)-p_{-1,1} \frac{1}{x}\left(\pi_{0, j-1}+x \pi_{1, j-1}\right)\right] \\
\varphi_{j}(x)=\frac{\left(p_{-1,0}+p_{-1,1}+p_{0,1}+p_{1,1}+p_{1,0}\right) \pi_{0, j}(x)-p_{-1,0} \frac{1}{x}\left(\pi_{0, j}+x \pi_{1, j}\right)-p_{0,1} \pi_{0, j-1}}{p_{1,0} x^{2}-\left(p_{-1,0}+p_{-1,1}+p_{0,1}+p_{1,1}+p_{1,0}\right) x+p_{-1,0}} \\
-\frac{p_{-1,1} \frac{1}{x}\left(\pi_{0, j-1}+x \pi_{1, j-1}\right)+\varphi_{j-1}(x)\left(p_{0,1}+p_{1,1} x+p_{-1,1} \frac{1}{x}\right)}{p_{1,0} x^{2}-\left(p_{-1,0}+p_{-1,1}+p_{0,1}+p_{1,1}+p_{1,0}\right) x+p_{-1,0}} .
\end{gathered}
$$

Then, using balance equation (2.24), we have

$$
\varphi_{j}(x)=\frac{p_{-1,0} \pi_{0, j}-p_{0,-1} \pi_{0, j+1} x-\varphi_{j-1}(x)\left(p_{0,1} x+p_{1,1} x^{2}+p_{1,1}\right)+p_{-1,1} \pi_{0, j-1}}{p_{1,0} x^{2}-\left(p_{-1,0}+p_{-1,1}+p_{0,1}+p_{1,1}+p_{1,0}\right) x+p_{-1,0}} .
$$

Based on the above results, we can determine $\varphi_{j}(x)$ recursively. 


\section{Proposition 3.2}

$$
\begin{aligned}
\varphi_{0}(x) & =\frac{\pi_{0,0}}{1-r_{0} x}, \\
\varphi_{j}(x) & =\frac{a_{j}}{1-r_{0} x}+\frac{r_{0}\left[p_{0,1}+p_{1,1}\left(x+x_{2}\right)\right]}{p_{1,0}\left(1-r_{0} x\right)}\left(\varphi_{j-1}(x)-\varphi_{j-1}\left(x_{2}\right)\right), \quad j \geq 1,
\end{aligned}
$$

where

$$
a_{j}=\frac{\bar{p} r \pi_{0, j+1} r_{0}}{p \bar{r}}
$$

and $\pi_{0,0}$ is given in equation (3.31).

Proof. For $(3.51)$, since $x_{2}(y)<1$ and $\psi_{0}(x)$ is analytic inside the unit circle, which implies $x_{2}(0)=\frac{r_{0}}{w}$ is also an zero of the numerator of the function on the right-hand side of (3.49). Therefore, (3.49) becomes

$$
\varphi_{0}(x)=\frac{\pi_{0,0}}{1-r_{0} x}
$$

where $r_{0}$ is given in (3.43) Similarly, for (3.52), based on the same argument, we have

$$
p_{-1,0} \pi_{0, \jmath}-p_{0,-1} \pi_{0, j+1} x_{2}-\varphi_{\jmath-1}\left(x_{2}\right)\left(p_{0,1} x_{2}+p_{1,1} x_{2}^{2}+p_{1,1}\right)+p_{-1,1} \pi_{0, j-1}=0 .
$$

Therefore,

$$
\begin{aligned}
\varphi_{\jmath}(x)= & \frac{p_{0,-1} \pi_{0, \jmath+1} x_{2}+\varphi_{\jmath-1}(x)\left(p_{0,1} x_{2}+p_{1,1} x_{2}^{2}+p_{-1,1}\right)-p_{-1,1} \pi_{0, \jmath-1}}{p_{1,0}\left(x-x_{1}\right)\left(x-x_{2}\right)} \\
& -\frac{p_{0,-1} \pi_{0, j+1} x+\varphi_{\jmath-1}(x)\left(p_{0,1} x+p_{1,1} x^{2}+p_{-1,1}\right)-p_{-1,1} \pi_{0, j-1}}{p_{1,0}\left(x-x_{1}\right)\left(x-x_{2}\right)}
\end{aligned}
$$




$$
\begin{aligned}
\varphi_{j}(x) & =\frac{p_{0,-1} \pi_{0, j+1} r_{0}}{p_{1,0}\left(1-r_{0} x\right)}+\frac{r_{0}\left[p_{0,1}\left(x-x_{2}\right)+p_{1,1}\left(x^{2}-x_{2}^{2}\right)\right]}{p_{1,0}\left(1-r_{0} x\right)} \frac{\left[\varphi_{j-1}(x)-\varphi_{j-1}\left(x_{2}\right)\right]}{x-x_{2}} \\
& =\frac{a_{j}}{1-r_{0} x}+\frac{r_{0}\left[p_{0,1}+p_{1,1}\left(x+x_{2}\right)\right]}{p_{1,0}\left(1-r_{0} x\right)}\left(\varphi_{j-1}(x)-\varphi_{j}\left(x_{2}\right)\right),
\end{aligned}
$$

where

$$
a_{j}=\frac{p_{0,-1} \pi_{0, j+1} r_{0}}{p_{1,0}}=\frac{\bar{p} r \pi_{0, j+1} r_{0}}{p \bar{r}}
$$

In the following, we determine the generating function $\psi_{0}(y)$.

Proposition 3.3 For $|y| \leq 1$, let

$$
\begin{aligned}
& T(y)=F(y)-\frac{\sqrt{\Delta(y)}}{2 a(y)}, \\
& F(y)=\frac{-b(y)}{2 a(y)}-y,
\end{aligned}
$$

where $a(y)$ and $b(y)$ are given in equation (3.34) and (3.35), respectively. Then,

$$
\psi_{0}(y)=\frac{x_{2}(y) \overline{p q} r(1-y) \pi_{0,0}}{(q y+\bar{q}) \bar{p} r} \frac{1}{T(y)}
$$

where $x_{2}(y)$ is the smaller zero of the key kernel function given in (3.38).

Proof. From the fundamental form for the generating function $P(x, y)$ given in (3.32), the key kernel equal to zero implies,

$$
\begin{aligned}
\psi_{0}(y) & =-\frac{H_{0}\left(x_{2}(y), y\right) \pi_{0,0}}{H_{2}\left(x_{2}(y), y\right)} \\
& =\frac{\overline{p q} r x_{2}(y)(1-y) \pi_{0,0}}{\overline{p q} r x_{2}(y)+\bar{p} q r x_{2}(y) y-\bar{p} q r y^{2}-\overline{p q} r y} \\
& =\frac{(1-y) x_{2}(y) \overline{p q} r \pi_{0,0}}{\bar{p} r\left[x_{2}(y)(\bar{q}+q)-q y^{2}-\bar{q} y\right]} \\
& =\frac{(1-y) x_{2}(y) \overline{p q} r \pi_{0,0}}{(q y+\bar{q}) \bar{p} r\left[x_{2}(y)-y\right]} .
\end{aligned}
$$


Substituting $F(y)$ and $T(y)$ into the above equation, we then have the final result for $\psi_{0}(y)$.

Lemma 3.5 For $\psi_{0}(y)=\frac{(1-y) x_{2}(y) \overline{p q} r \pi_{0,0}}{(q y+\bar{q}) \bar{p} r\left[x_{2}(y)-y\right]}, \frac{x_{2}(y)}{q y+\bar{q}}$ is analytic in the cut plane $C_{y}-$ $\left[y_{3}, y_{4}\right]$.

Proof. It is easy to see that the only possible pole of $\frac{x_{2}(y)}{q y+\bar{q}}$ is $-\frac{\bar{q}}{q}$. But,

$$
\begin{aligned}
\frac{x_{2}(y)}{q y+\bar{q}} & =\frac{-b(y)-\sqrt{\Delta(y)}}{2 a(y)(q y+\bar{q})}=\frac{b^{2}(y)-\Delta(y)}{2 a(y)(q y+\bar{q})[-b(y)+\sqrt{\Delta(y)}]} \\
& =\frac{2 c(y)}{(q y+\bar{q})[-b(y)+\sqrt{\Delta(y)}]}=\frac{2 \bar{p} r}{-b(y)+\sqrt{\Delta(y)}}
\end{aligned}
$$

implies that $\frac{x_{2}(y)}{q y+\bar{q}}$ is analytic at $y=-\frac{\bar{q}}{q}$ since $-b\left(-\frac{\bar{q}}{q}\right)+\sqrt{\Delta\left(-\frac{\bar{q}}{q}\right)}=-2 b\left(-\frac{\bar{q}}{q}\right)>0$.

Lemma 3.5 implies that the possible poles of $\psi_{0}(y)$ are zero of $x_{2}(y)-y$. Since $x_{2}\left(-\frac{\vec{q}}{q}\right)=0\left(\right.$ note $\left.x_{2}(y)=\frac{2 c(y)}{-b(y)+\sqrt{\Delta(y)}}\right), y=-\frac{\bar{q}}{q}$ is not a root of $x_{2}(y)-y$. So assuming $y \neq-\frac{\bar{q}}{q}, x_{2}(y)-y=0$ implies

$$
\begin{aligned}
-b(y)-2 a(y) y & =\sqrt{\Delta(y)} \\
b^{2}(y)+4 b(y) a(y) y+4 a^{2}(y) y^{2} & =b^{2}(y)-4 a(y) c(y), \\
a(y) y^{2}+b(y) y+c(y) & =0 .
\end{aligned}
$$

Hence, a zero of $x_{2}(y)-y$ has to be a zero of $K_{0}(y, y)=a(y) y^{2}+b(y) y+c(y)$. Also, since $y_{1}$ is the smaller branch point of the key kernel function, $x_{2}\left(y_{1}\right)-y_{1}=$ $\frac{-b\left(y_{1}\right)}{2 a\left(y_{1}\right)}-y_{1}=F\left(y_{1}\right)$. So, the possible poles of $\psi_{0}(y)$ are the zeros of $F\left(y_{1}\right)$ and equivalently, the zeros of $K_{0}(y, y)=a(y) y^{2}+b(y) y+c(y)$.

Now we determine the zeros of $K_{0}(y, y)=a(y) y^{2}+b(y) y+c(y)$. 
Proposition 3.4 The key kernel $K_{0}(y, y)=a(y) y^{2}+b(y) y+c(y)$ can be written in the form: $K_{0}(y, y)=(y-1)\left(y-\eta_{1}\right)\left(y-\eta_{2}\right)$, and the two non-zero roots are:

$$
\begin{aligned}
& \eta_{1}=\frac{-B+\sqrt{B^{2}-4 A C}}{2 A}, \\
& \eta_{2}=\frac{-B-\sqrt{B^{2}-4 A C}}{2 A},
\end{aligned}
$$

where

$$
\begin{aligned}
& A=p q \bar{r} \\
& B=p \bar{r}+(\overline{p r}+p r) q, \\
& C=-\overline{p q} r .
\end{aligned}
$$

We also have $\eta_{1}>0, \eta_{2}<0$ and $\left|\eta_{2}\right|>\eta_{1}$.

\section{Proof.}

$$
\begin{aligned}
K_{0}(y, y) & =a(y) y^{2}+b(y) y+c(y) \\
& =p \bar{r}(q y+\bar{q}) y^{2}+[(\overline{p r}+p r)(q y+\bar{q})-1] y+\bar{p} r(q y+\bar{q}) \\
& =(y-1)\left(A y^{2}+B y+C\right)=(y-1)\left(y-\eta_{1}\right)\left(y-\eta_{2}\right) \\
& =a(y)\left(x_{1}(y)-y\right)\left(x_{2}(y)-y\right)
\end{aligned}
$$

The long division method is used to get $A, B$ and $C$. It is clear that $A>0, B>0$ and $C<0$. Then, it is obvious that $\eta_{1}>0, \eta_{2}<0$ and $\left|\eta_{2}\right|>\eta_{1}$ can be proved using simple algebra. By the quadratic formula, we obtain two roots of $K_{0}(y, y)$. This completes the proof. 
Let

$$
\begin{aligned}
T(y) & =x_{2}(y)-y, \\
T^{*}(y) & =a(y)\left(x_{1}(y)-y\right) .
\end{aligned}
$$

So $K_{0}(y, y)=T^{*}(y) T(y)$. We have $T^{*}(0)=a(0) x_{1}(0)=\frac{-b(0)+\sqrt{\Delta(0)}}{2}>0$ since $b(0)<0$. We also know $T^{*}(-\infty) \rightarrow-\infty$ as $y \rightarrow-\infty$. These two conditions imply that $T^{*}(y)$ has a root in the interval $(0,-\infty)$, which has to be $\eta_{2}$ since $\eta_{2}<0$. Hence we can conclude that the only possible pole of $\psi_{0}(y)$ is $\eta_{1}$.

As will be seen in the later chapter (from the Key Lemma), the type of exact tail asymptotics relies on the region defined by the value of $F\left(y_{1}\right)$ at the branch point $y_{1}$. Now we first calculate $F\left(y_{1}\right)$.

\section{Corollary 3.2}

$$
F\left(y_{1}\right)=\frac{2 \sqrt{\overline{p r} p r}}{(\overline{p r}-p r)^{2} q}
$$

PROOF.

$$
\begin{aligned}
F\left(y_{1}\right) & =-\frac{b\left(y_{1}\right)}{2 a\left(y_{1}\right)}-y_{1} \\
& =\frac{x_{1}\left(y_{1}\right)+x_{2}\left(y_{1}\right)}{2}-y_{1} \\
& =\frac{\overline{p r}+p r}{(\overline{p r}+p r)^{2}}-\frac{\bar{q}}{q}-\frac{\overline{p r}+p r-2 \sqrt{\overline{p r} p r}}{(\overline{p r}-p r)^{2} q}+\frac{\bar{q}}{q} \\
& =\frac{2 \sqrt{\overline{p r} p r}}{(\overline{p r}-p r)^{2} q} .
\end{aligned}
$$


We can divide the whole stability region into three parts, as $F\left(y_{1}\right)>0, F\left(y_{1}\right)=0$ and $F\left(y_{1}\right)<0$.

Also we have the following equivalent stability condition.

Lemma 3.6 $\rho=\rho_{h}+\rho_{l}<1$, if and only if $T^{\prime}(1)<0$.

Proof. Since $T(y)=x_{2}(y)-y$, we just need to prove $T^{\prime}(1)=x_{2}^{\prime}(1)-1<0$.

From the kernel equation $K_{0}(x, y)=a(y) x^{2}+b(y) x+c(y)$, we know

$$
\begin{aligned}
x_{2}(y)+x_{1}(y) & =-\frac{b(y)}{a(y)}, \\
x_{2}(y) x_{1}(y) & =\frac{c(y)}{a(y)} .
\end{aligned}
$$

Taking derivatives for above two equations to have,

$$
\begin{aligned}
x_{2}^{\prime}(y)+x_{1}^{\prime}(y) & =-\frac{b^{\prime}(y) a(y)-a^{\prime}(y) b(y)}{a(y)^{2}}=A(y), \\
x_{2}^{\prime}(y) x_{1}(y)+x_{1}^{\prime}(y) x_{2}(y) & =\frac{c^{\prime}(y) a(y)-a^{\prime}(y) c(y)}{a(y)^{2}}=B(y) .
\end{aligned}
$$

where

$$
\begin{aligned}
& A(y)=-\frac{b^{\prime}(y) a(y)-a^{\prime}(y) b(y)}{a(y)^{2}}, \\
& B(y)=\frac{c^{\prime}(y) a(y)-a^{\prime}(y) c(y)}{a(y)^{2}} .
\end{aligned}
$$

Solve for $x_{2}^{\prime}(y)$,

$$
\begin{aligned}
& x_{2}^{\prime}(y)=\frac{B(y)-A(y) x_{2}(y)}{x_{1}(y)-x_{2}(y)}, \\
& x_{2}^{\prime}(1)=\frac{B(1)-A(1) x_{2}(1)}{x_{1}(1)-x_{2}(1)},
\end{aligned}
$$


where

$$
\begin{aligned}
A(1) & =-\frac{(\overline{p r}+p r) p q \bar{r}-p q \bar{r}(\overline{p r}+p r-1)}{(p \bar{r})^{2}}=-\frac{q}{p \bar{r}}, \\
B(1) & =\frac{\overline{p r} p q r-\overline{p r} p q r}{(p \bar{r})^{2}}=0, \\
x_{2}(1) & =\frac{-b(1)-\sqrt{\Delta(1)}}{2 a(1)} \\
x_{2}(1)-x_{1}(1) & =-\frac{\sqrt{\Delta(1)}}{a(1)} .
\end{aligned}
$$

Hence,

$$
x_{2}^{\prime}(1)=\frac{q}{2 p \tilde{r}}\left(\frac{b(1)}{\sqrt{\Delta(1)}}+1\right)
$$

since we know $a(1)+b(1)+c(1)=0$,

$$
\begin{aligned}
\Delta(1) & =b^{2}(1)-4 a(1) c(1)=(a(1)-c(1))^{2}=(p \bar{r}-\bar{p} r)^{2} \\
\sqrt{\Delta(1)} & =|p \bar{r}-\bar{p} r|
\end{aligned}
$$

Since we assume $\rho_{h}=\frac{p}{r}<1$, we have $p<r$, also $\bar{r}<\bar{p}$. Hence, we have $p \bar{r}<r \bar{p}$.

This leads to

$$
\sqrt{\Delta(1)}=\bar{p} r-p \bar{r}
$$

Hence, we have

$$
x_{2}^{\prime}(1)=\frac{q}{2 p \bar{r}}\left(\frac{\overline{p r}+p r-1}{\bar{p} r-p \bar{r}}+1\right) .
$$


To prove $x_{2}^{\prime}(1)-1<0$, we just need to prove

$$
\begin{aligned}
\frac{q}{2 p \bar{r}}\left(\frac{\overline{p r}+p r-1}{\bar{p} r-p \bar{r}}+1\right) & <1, \\
\frac{\overline{p r}+p r-1}{\bar{p} r-p \bar{r}} & <\frac{2 p \bar{r}}{q}-1, \\
\frac{(\overline{p r}+p r-1) q-(\bar{p} r-p \bar{r})(2 p \bar{r}-q)}{(\bar{p} r-p \bar{r}) q} & <0 .
\end{aligned}
$$

The denominator is obviously nonnegative, we just need to prove,

$$
\begin{aligned}
(\overline{p r}+p r-1) q-(\bar{p} r-p \bar{r})(2 p \bar{r}-q) & <0, \\
\overline{p r} q+p r q-q-2 p r \overline{p r}+\bar{p} q r+2(p \bar{r})^{2}+p q \bar{r} & <0 \\
q \bar{r}+q r-q-2 p \bar{r}(\bar{p} r-p \bar{r}) & <0, \\
-2 p \bar{r}(\bar{p} r-p \bar{r}) & <0,
\end{aligned}
$$

which is true, since $p \bar{r}<r \bar{p}$ as shown previously. This completes the proof. $T^{\prime}(1)<0$ means $T^{\prime}(y)$ is a decreasing function at $x=1$. 


\section{Chapter 4}

\section{Analysis of Singularities}

This chapter focuses on the analysis of singularities of the generating function $\psi_{0}(y)$ since the exact tail asymptotics along the low-priority queue direction relies on the singularities analysis. The asymptotics of the coefficients of $\psi_{0}(y)$ are obtained using a Tauberian-like theorem, such as Theorem 4 in Bender [4] or Corollary 2 in Flajolet and Odlyzko [15]. The theorem is restated in the following lemma for convenience.

Lemma 4.1 (Flajolet and Odlyzko)A ssume that $f(z)$ is analytıc in $\Delta(\phi, \epsilon)=\{z$ : $|z|<1+\epsilon,|\operatorname{Arg}(z-1)| \geq \phi, \epsilon>0,0<\phi<\pi / 2\}$, except at $z=1$ and

$$
f(z) \sim K(1-z)^{s} \text { as } z \rightarrow 1 \text { in } \Delta(\phi, \epsilon) .
$$

Then, as $n \rightarrow \infty:$ ( ( ) If $s \notin\{0,1,2, \ldots\}$,

$$
f_{n} \sim \frac{K}{\Gamma(-s)} n^{-s-1}
$$


(ii) If $s$ is a nonnegative integer, then

$$
f_{n}=o\left(n^{-s-1}\right) .
$$

We use the the following notation in this paper. For a function $f(y)$ that is analytic at $y=0$, we denote the coefficient of $y^{k}$ in the Taylor expression of $f(y)$ by $C_{k}[f(y)]$.

Our goal is to locate the dominant singularity, which determine the decay for tail asymptotics. The following key lemma plays a key role in asymptotic analysis.

Lemma 4.2 (Key Lemma) For the property of $\eta_{1}$, there are three cases:

(i) If $F\left(y_{1}\right)>0$, then $\eta_{1}$ is a zero of $T(y)$ and $\eta_{1}<y_{1}$. Hence $\eta_{1}$ is the dominant singular point of $\psi_{0}(y)$, which is a pole.

(ii) If $F\left(y_{1}\right)=0$, then $\eta_{1}$ is a zero of $T(y)$ and $\eta_{1}=y_{1}$. Hence $\eta_{1}=y_{1}$ is the dominant singular point of $\psi_{0}(y)$, which is a branch point.

(iii) If $F\left(y_{1}\right)<0$, then $\eta_{1}$ is a zero of $T^{*}(y)$. Hence $\psi_{0}(y)$ has no poles and $y_{1}$ is the dominant singular point, which is a branch point.

Proof. Since $y_{1}$ is the zero of $\Delta(y)$, then from equation (3.55) it is clear that $T\left(y_{1}\right)>0,=0$, or $<0$ is equivalent to $F\left(y_{1}\right)>0,=0$ or $<0$, respectively. For case (i): From lemma 3.6, we know $T^{\prime}(1)<0$. We can also show $T(1)=0$ from the following steps. To prove $T(1)=0$ we just need to show $x_{2}(1)=1$. This can be simplified to the following, (simple algebra is used):

$$
\begin{aligned}
\frac{-b(1)-\sqrt{\Delta(1)}}{2 a(1)} & =1, \\
-b(1)-2 a(1) & =\sqrt{\Delta(1)} .
\end{aligned}
$$


and

$$
-b(1)-2 a(1)=-\overline{p r}-p r+1-2 p \bar{r}=-\bar{r}-p+1=r-p>0
$$

since we assume $\rho_{h}=\frac{p}{r}<1$. Hence $-b(1)-2 a(1)$ is nonnegative. Then, squaring both sides of equation, we have

$$
\begin{aligned}
b(1)^{2}+4 a(1)^{2}+4 a(1) b(1) & =b(1)^{2}-4 a(1) c(1), \\
a(1)+b(1) & =-c(1) .
\end{aligned}
$$

The last step is obviously true since we have $a(1)+b(1)+c(1)=0$. Hence, $T(y)$ has a root at $y=1$. From the above conditions, we can conclude there exists a number $y^{\prime}$ with $1<y^{\prime}<y_{1}$, such that $T\left(y^{\prime}\right)<0$. Then, by the Mean Value Theorem from calculus, we know $T(y)$ has a root in the interval $\left(1, y_{1}\right)$, which has to be $\eta_{1}$.

For case (ii): If $F\left(y_{1}\right)=0$, we have $T\left(y_{1}\right)=F\left(y_{1}\right)=0$. And also from Lemma 3.6 and $T(1)=0$, these conditions yield that $y_{1}=\eta_{1}$.

For case (iii): If $F\left(y_{1}\right)<0$, we have $T^{*}\left(y_{1}\right)=a\left(y_{1}\right) F\left(y_{1}\right)<0$, since $a\left(y_{1}\right)>0$. We can also show $T^{*}(1)>0$. We know $T^{*}(1)=a(1)\left(x_{1}(1)-1\right)$. To show $T^{*}(1)>0$, we just need to prove $x_{1}(1)-1>0$ since $a(1)>0$. This could be simplified to the following steps:

$$
\begin{aligned}
x_{1}(1)-1 & >0 \\
\frac{-b(1)+\sqrt{\Delta(1)}}{2 a(1)}-1 & >0 \\
-b(1)+\sqrt{\Delta(1)}-2 a(1) & >0
\end{aligned}
$$




$$
\begin{aligned}
-\overline{p r}-p r+1+\bar{p} r-p \bar{r}-2 p \bar{r} & >0 \\
r-p+\bar{p} r-p \bar{r} & >0 .
\end{aligned}
$$

This is obviously true from assumption $\rho_{h}=\frac{p}{r}<1$, and $\bar{p} r-p \bar{r}>0$ has shown previously. Hence, it follows that $T^{*}(y)$ has a root between 1 and $y_{1}$, which has to be $\eta_{1}$.

The three cases of $F\left(y_{1}\right)$ in the key lemma corresponding to the three types of exact tail asymptotics along the low-priority queue direction as shown later: (i) exact geometric; (ii) geometric with a pre-factor $n^{-\frac{1}{2}}$; and (iii) geometric with a pre-factor $n^{-\frac{3}{2}}$. 


\section{Chapter 5}

\section{Exact Tail Asymptotics for the Low-Priority Queue}

In this chapter, we express the proof of Theorem 1.2. To characterize the tail asympotoics in $\pi_{i, j}$ for fixed $i \geq 0$, we analyze the generating function of $\psi_{0}(y)$ and $\psi_{i}(y)$. The proof is done in two parts. First, for case $i=0$, we characterize the exact tail asymptotics for $\pi_{0, j}$. Then, we find an expression of $\psi_{i}(y)$ using $\psi_{0}(y)$ and based on the result of $\pi_{0, j}$, we take the case $i>0$, and express the tail asymptotics of $\pi_{i, j}$.

\subsection{Exact Tail Asymptotics of $\pi_{0, j}$}

This is the case of $i=0$. Based on the Key Lemma from 4.2, we have the following:

Lemma 5.1 For the discrete-time preemptive priority queue with two classes of customers satisfying $\rho<1$, characterizations of the exact asymptotics in the joint stationary distribution along the low-priority queue direction are given below for 
zero number of high-priority customers, with $F\left(y_{1}\right)$ given in (3.64):

(i) In the region defined by $F\left(y_{1}\right)>0$,

$$
\pi_{0, j} \sim C_{l, 1}\left(\frac{1}{\eta_{1}}\right)^{j}
$$

where

$$
C_{l, 1}=\frac{x_{2}\left(\eta_{1}\right) \overline{p q} r\left(\eta_{1}-1\right) \pi_{0,0}}{\eta_{1}\left(q \eta_{1}+\bar{q}\right) \bar{p} r} \frac{1}{T^{\prime}\left(\eta_{1}\right)}
$$

with $\pi_{0,0}$ given by (3.31). (ii) In the region defined by $F\left(y_{1}\right)=0$,

$$
\pi_{0, j} \sim C_{l, 2} j^{-1 / 2}\left(\frac{1}{y_{1}}\right)^{j}
$$

where

$$
C_{l, 2}=\frac{2 x_{2}\left(y_{1}\right) \overline{p q} r\left(y_{1}-1\right) \pi_{0,0} p \bar{r}}{\sqrt{\pi} \bar{p} r \sqrt{d\left(1-\frac{y_{1}}{y_{2}}\right)}}
$$

with d given by equation (3.48).

(ini) In the region defined by $F\left(y_{1}\right)<0$,

$$
\pi_{0,3} \sim C_{l, 3} j^{-3 / 2}\left(\frac{1}{y_{1}}\right)^{\jmath}
$$

where

$$
\begin{aligned}
C_{l, 3}= & \frac{x_{2}\left(y_{1}\right) \overline{p q} r \pi_{0,0} \bar{p} r}{4 \bar{p}[\bar{p} r(x-y)]^{2}(q y+\bar{q})^{4}}\left[\left(q y_{1}+\bar{q}\right)\left(x_{2}\left(y_{1}\right)-y_{1}\right)-\left(q x_{2}\left(y_{1}\right)-2 q y_{1}-\bar{q}\right)\left(1-y_{1}\right)\right] \\
& \left\{\frac{\sqrt{d}\left(y_{1}-y_{2}\right)\left(q y_{1}+\bar{q}\right)}{\sqrt{\left(1-\frac{y_{1}}{y_{2}}\right)}}+2 q \sqrt{\frac{\Delta\left(y_{1}\right)}{d}}\left(1-\sqrt{\Delta\left(y_{1}\right)}\right)\right\}
\end{aligned}
$$


Proof. For case (i): In this case, it follows that

$$
\begin{aligned}
\left(1-\frac{y}{\eta_{1}}\right) \psi_{0}(y) & =\left(-\frac{y-\eta_{1}}{\eta_{1}}\right) \frac{x_{2}(y) \bar{p} \bar{q} r(1-y) \pi_{0,0}}{(q y+\bar{q}) \bar{p} r T(y)} \\
& =-\frac{x_{2}(y) \overline{p q} r(1-y) \pi_{0,0}}{\eta_{1}(q y+\bar{q}) \bar{p} r} \frac{\left(y-\eta_{1}\right)}{T(y)} \\
& =-\frac{x_{2}(y) \overline{p q} r(1-y) \pi_{0,0}}{\eta_{1}(q y+\bar{q}) \bar{p} r} \frac{1}{\frac{T(y)-T\left(\eta_{1}\right)}{y-\eta_{1}}}
\end{aligned}
$$

In the last step, we used $T\left(\eta_{1}\right)=x_{2}\left(\eta_{1}\right)-\eta_{1}=0$ since from the key lemma, in this case $\eta_{1}$ is a zero of $T(y)$. So,

$$
\begin{aligned}
\lim _{\frac{\underline{y}}{\eta_{1}} \rightarrow 1}\left(1-\frac{y}{\eta_{1}}\right) \psi_{0}(y) & =-\frac{x_{2}\left(\eta_{1}\right) \overline{p q} r\left(1-\eta_{1}\right) \pi_{0,0}}{\eta_{1}\left(q \eta_{1}+\bar{q}\right) \bar{p} r} \frac{1}{T^{\prime}\left(\eta_{1}\right)} \\
& =\frac{x_{2}\left(\eta_{1}\right) \overline{p q} r\left(\eta_{1}-1\right) \pi_{0,0}}{\eta_{1}\left(q \eta_{1}+\bar{q}\right) \bar{p} r} \frac{1}{T^{\prime}\left(\eta_{1}\right)}=C_{l, 1} .
\end{aligned}
$$

In order to get the asymptotics for $\pi_{0, j}$, we write $\psi_{0}(y)$ as the following form:

$$
\psi_{0}(y)=\sum_{j=0}^{\infty} \pi_{0, j} y^{j}=\sum_{j=0}^{\infty}\left(\eta_{1}\right)^{j} \pi_{0, j}\left(\frac{y}{\eta_{1}}\right)^{j}=\sum_{j=0}^{\infty} f_{j} z^{j}
$$

where $f_{j}=\left(\eta_{1}\right)^{j} \pi_{0, j}$ and $z=\frac{y}{\eta_{1}}$. It is obvious that $\psi_{0}(y)$ is analytic in the region $\Delta(\phi, \epsilon)=\left\{y:\left|\frac{y}{\eta_{1}}\right| \leq 1+\epsilon,\left|\operatorname{Arg}\left(\frac{y}{\eta_{1}}-1\right)\right| \geq \phi, \epsilon>0,0<\phi<\pi / 2\right\}$ except at $\frac{y}{\eta_{1}}=1$. Then we can use Theorem 4.1 on $\psi_{0}(y)$ with $s=-1, K=C_{l, 1}$ to have

$$
C_{j}\left[\psi_{0}(y)\right] \sim \frac{C_{1,1}}{\Gamma(1)} j^{1-1} \sim C_{l, 1}
$$

Hence, we have

$$
\pi_{0, j} \sim C_{l, 1}\left(\frac{1}{\eta_{1}}\right)^{\jmath}
$$


which leads to the result of $\pi_{0, \jmath}$ in case (i).

For case (ii): In this case, from equation (3.55),

$$
\frac{1}{T(y)}=\frac{2 a(y)}{-b(y)-2 a(y) y-\sqrt{\Delta(y)}}=\frac{2 a(y)}{F^{*}(y)-\sqrt{\Delta(y)}},
$$

where $F^{*}(y)=-b(y)-2 a(y) y$. We have $F^{*}\left(y_{1}\right)=-2 a\left(y_{1}\right) F\left(y_{1}\right)=0$ since $F\left(y_{1}\right)=0$,. Then, using equation (3.47), we have the following:

$$
\begin{aligned}
F^{*}(y)-\sqrt{\Delta(y)} & =\left[F^{*}(y)-F^{*}\left(y_{1}\right)\right]-\sqrt{d\left(1-\frac{y}{y_{1}}\right)\left(1-\frac{y}{y_{2}}\right)} \\
& =\left(y-y_{1}\right) \frac{F^{*}(y)-F^{*}\left(y_{1}\right)}{y-y_{1}}-\sqrt{d\left(1-\frac{y}{y_{1}}\right)\left(1-\frac{y}{y_{2}}\right)} \\
& =\sqrt{1-\frac{y}{y_{1}}}\left\{\sqrt{1-\frac{y}{y_{1}}} \frac{\left(F^{*}(y)-F^{*}\left(y_{1}\right)\right)}{y-y_{1}}\left(-y_{1}\right)-\sqrt{d\left(1-\frac{y}{y_{2}}\right)}\right\},
\end{aligned}
$$

where $d$ is given in (3.48).

It follows that

$$
\begin{aligned}
\sqrt{1-\frac{y}{y_{1}}} \psi_{0}(y) & =\frac{x_{2}(y) \overline{p q} r(1-y) \pi_{0,0}}{(q y+\bar{q}) \bar{p} r} \frac{1}{T(y)} \sqrt{1-\frac{y}{y_{1}}} \\
& =\frac{2 x_{2}(y) \overline{p q} r(1-y) \pi_{0,0} p \bar{r} \sqrt{1-\frac{y}{y_{1}}}}{\bar{p} r \sqrt{1-\frac{y}{y_{1}}}\left\{\sqrt{1-\frac{y}{y_{1}} \frac{F^{*}(y)-F^{*}\left(y_{1}\right)}{y-y_{1}}\left(-y_{1}\right)-\sqrt{d\left(1-\frac{y}{y_{2}}\right)}}\right\}} \\
& =\frac{2 x_{2}(y) \bar{p} \bar{q} r(1-y) \pi_{0,0} p \bar{r}}{\bar{p} r\left\{\sqrt{1-\frac{y}{y_{1}}} \frac{F^{*}(y)-F^{*}\left(y_{1}\right)}{y-y_{1}}\left(-y_{1}\right)-\sqrt{d\left(1-\frac{y}{y_{2}}\right)}\right\}} .
\end{aligned}
$$


Hence,

$$
\begin{aligned}
& \lim _{\frac{y}{y_{1}} \rightarrow 1} \sqrt{1-\frac{y}{y_{1}}} \psi_{0}(y) \\
= & \lim _{\frac{y}{y_{1}} \rightarrow 1} \frac{2 x_{2}(y) \overline{p q} r(1-y) \pi_{0,0} p \bar{r}}{\bar{p} r\left\{\sqrt{\left.1-\frac{y}{y_{1}} \frac{F^{*}(y)-F^{*}\left(y_{1}\right)}{y-y_{1}}\left(-y_{1}\right)-\sqrt{d\left(1-\frac{y}{y_{2}}\right)}\right\}}\right.} \\
= & \frac{2 x_{2}\left(y_{1}\right) \overline{p q} r\left(y_{1}-1\right) \pi_{0,0} p \bar{r}}{\bar{p} r \sqrt{d\left(1-\frac{y_{1}}{y_{2}}\right)}}=C_{l, 2}^{*} .
\end{aligned}
$$

Similarly to case (i), in order to get the asymptotics for $\pi_{0, \jmath}$, we rewrite $\psi_{0}(y)$ :

$$
\psi_{0}(y)=\sum_{\jmath=0}^{\infty} \pi_{0, \jmath} y^{\jmath}=\sum_{\jmath=0}^{\infty}\left(y_{1}\right)^{\jmath} \pi_{0, \jmath}\left(\frac{y}{y_{1}}\right)^{\jmath}=\sum_{\jmath=0}^{\infty} f_{\jmath} z^{\jmath}
$$

where $f_{\jmath}=\left(y_{1}\right)^{\jmath} \pi_{0, \jmath}$ and $z=\frac{y}{y_{1}}$. It is obvious that $\psi_{0}(y)$ is analytic in the region $\Delta(\phi, \epsilon)=\left\{y:\left|\frac{y}{y_{1}}\right| \leq 1+\epsilon,\left|\operatorname{Arg}\left(\frac{y}{y_{1}}-1\right)\right| \geq \phi, \epsilon>0,0<\phi<\pi / 2\right\}$ except at $\frac{y}{y_{1}}=1$. Then using Theorem 4.1 on $\psi_{0}(y)$ with $s=-\frac{1}{2}$ and $K=C_{1,2}^{*}$ we have

$$
C_{\jmath}\left[\psi_{0}(y)\right] \sim \frac{C_{l, 2}^{*}}{\Gamma\left(\frac{1}{2}\right)} \jmath^{-\frac{1}{2}} \sim C_{l, 2} \jmath^{-\frac{1}{2}}
$$

where

$$
C_{l, 2}=\frac{C_{l, 2}^{*}}{\sqrt{\pi}}
$$

Hence, we have

$$
\pi_{0,3} \sim C_{l, 2} \jmath^{-\frac{1}{2}}\left(\frac{1}{y_{1}}\right)^{\jmath}
$$


which leads to the result of $\pi_{0, J}$ in case (ii).

For case (iii): In this case, $T(y)$ has no zeros. We take the derivative of $\psi_{0}(y)$. To make things easier, let

$$
G(x, y)=\frac{x \overline{p q} r(1-y) \pi_{0,0}}{(q y+\bar{q}) \bar{p} r(x-y)}
$$

then $\psi_{0}(y)=G\left(x_{2}(y), y\right)$. So

$$
\psi_{0}^{\prime}(y)=\frac{\partial G}{\partial x}+\frac{\partial G}{\partial y} \frac{d x_{2}(y)}{d y}
$$

We have following calculations:

$$
\begin{aligned}
\frac{\partial G}{\partial x}= & \frac{\overline{p q} r(1-y) \pi_{0,0}(q y+\bar{q}) \bar{p} r(x-y)-(q y+\bar{q}) \bar{p} r x \overline{p q} r(1-y) \pi_{0,0}}{[(q y+\bar{q}) \bar{p} r(x-y)]^{2}} \\
= & \frac{\bar{p} \bar{q} r(y-1) \pi_{0,0} y}{(q y+\bar{q}) \bar{p} r(x-y)^{2}}, \\
\frac{\partial G}{\partial y}= & \frac{-x \overline{p q} r \pi_{0,0}(q y+\bar{q}) \bar{p} r(x-y)-\bar{p} r(q x-2 q y-\bar{q}) x \bar{p} r(1-y) \pi_{0,0}}{[(q y+\bar{q}) \bar{p} r(x-y)]^{2}} \\
= & -x \bar{p} \bar{q} r \pi_{0,0} \bar{p} r\left\{\frac{(q y+\bar{q})(x-y)-(q x-2 q y-\bar{q})(1-y)}{[(q y+\bar{q}) \bar{p} r(x-y)]^{2}}\right\}, \\
\frac{d x_{2}(y)}{d y}= & \frac{1}{[2 p \bar{r}(q y+\bar{q})]^{2}}\left\{\left[-(\overline{p r}+p r) q-\frac{1}{2} \Delta(y)^{-1 / 2} \Delta(y)^{\prime}\right] 2 p \bar{r}(q y+\bar{q})\right. \\
= & \frac{1}{2 p \bar{r}(q y+\bar{q})^{2}}\left\{\left[-(\overline{p r}+p r) q-\frac{\sqrt{d}\left[2 y-\left(y_{1}+y_{2}\right)\right]}{\left.2 \sqrt{\left(1-\frac{y}{y_{1}}\right)\left(1-\frac{y}{y_{2}}\right)}\right]}\right](q y+\bar{q})\right. \\
& \left.-\left[-(\overline{p r}+p r)(q y+\bar{q})+1-\sqrt{d\left(1-\frac{y}{y_{1}}\right)\left(1-\frac{y}{y_{2}}\right)}\right] q\right\} .
\end{aligned}
$$


From equation (3.47),

$$
\begin{aligned}
\Delta(y)^{\prime} & =\frac{d}{y_{1} y_{2}}\left(2 y-\left(y_{1}+y_{2}\right)\right) \\
\frac{d x_{2}(y)}{d y} & =\frac{1}{2 p \bar{r}(q y+\bar{q})^{2}}\left\{-\frac{\sqrt{d}\left(2 y-\left(y_{1}+y_{2}\right)\right)(q y+\bar{q})}{2 \sqrt{\left(1-\frac{y}{y_{1}}\right)\left(1-\frac{y}{y_{2}}\right)}}-q+q \sqrt{d\left(1-\frac{y}{y_{1}}\right)\left(1-\frac{y}{y_{2}}\right)}\right\} .
\end{aligned}
$$

Then it follows that

$$
\begin{aligned}
& \lim _{\frac{y}{y_{1}} \rightarrow 1} \sqrt{\left(1-\frac{y}{y_{1}}\right)} \psi_{0}(y)^{\prime} \\
& =\lim _{\frac{y}{y_{1}} \rightarrow 1} \sqrt{\left(1-\frac{y}{y_{1}}\right)} \frac{\partial G}{\partial x}+\lim _{\frac{y}{y_{1}} \rightarrow 1} \sqrt{\left(1-\frac{y}{y_{1}}\right)} \frac{\partial G}{\partial y} \frac{d x_{2}(y)}{d y} \\
& =\lim _{\frac{y}{y_{1}} \rightarrow 1} \sqrt{\left(1-\frac{y}{y_{1}}\right)} \frac{\partial G}{\partial y} \frac{d x_{2}(y)}{d y} \\
& =\lim _{\frac{y}{y_{1}} \rightarrow 1} \frac{x \overline{p q} r \pi_{0,0} \bar{p} r}{2 p \bar{r}[\bar{p} r(x-y)]^{2}(q y+\bar{q})^{4}} \sqrt{\left(1-\frac{y}{y_{1}}\right)}[(q y+\bar{q})(x-y)-(q x-2 q y-\bar{q})(1-y)] \\
& \left\{\frac{\sqrt{d}(q y+\bar{q})\left[2 y-\left(y_{1}+y_{2}\right)\right]}{2 \sqrt{\left(1-\frac{y}{y_{1}}\right)\left(1-\frac{y}{y_{2}}\right)}}+q-q \sqrt{d\left(1-\frac{y}{y_{1}}\right)\left(1-\frac{y}{y_{2}}\right)}\right\} \\
& =\frac{x_{2}\left(y_{1}\right) \bar{p} \bar{q} r \pi_{0,0} \bar{p} r}{4 p \bar{r}[\bar{p} r(x-y)]^{2}(q y+\bar{q})^{4}}\left[\left(q y_{1}+\bar{q}\right)\left(x_{2}\left(y_{1}\right)-y_{1}\right)-\left(q x_{2}\left(y_{1}\right)-2 q y_{1}-\bar{q}\right)\left(1-y_{1}\right)\right] \\
& \left\{\frac{\sqrt{d}\left(y_{1}-y_{2}\right)\left(q y_{1}+\bar{q}\right)}{\sqrt{\left(1-\frac{y_{1}}{y_{2}}\right)}}+2 q \sqrt{\frac{\Delta\left(y_{1}\right)}{d}}\left(1-\sqrt{\Delta\left(y_{1}\right)}\right)\right\} \\
& =C_{l, 3}^{*} \text {. }
\end{aligned}
$$


In order to get the asymptotics for $\pi_{0, j}$, we rewrite $\psi_{0}^{\prime}(y)$ as the following:

$$
\begin{aligned}
\psi_{0}^{\prime}(y) & =\sum_{j=0}^{\infty} j \pi_{0, j} y^{j-1}=\sum_{j=0}^{\infty}(j+1) \pi_{0, j+1} y^{3} \\
& =\sum_{j=0}^{\infty}(j+1) \pi_{0, j+1} y_{1}^{j}\left(\frac{y}{y_{1}}\right)^{j} \\
& =\sum_{j=0}^{\infty} f_{j+1} z^{\jmath}
\end{aligned}
$$

where $f_{j+1}=(j+1) \pi_{0, j+1} y_{1}^{j}$, and $z=\frac{y}{y_{1}}$. It is obvious that $\psi_{0}(y)$ is analytic in the region $\Delta(\phi, \epsilon)=\left\{y:\left|\frac{y}{y_{1}}\right| \leq 1+\epsilon,\left|\operatorname{Arg}\left(\frac{y}{y_{1}}-1\right)\right| \geq \phi, \epsilon>0,0<\phi<\pi / 2\right\}$ except at $\frac{y}{y_{1}}=1$. Then use Theorem 4.1 on $\psi_{0}^{\prime}(y)$ with $s=-\frac{1}{2}$ and $K=C_{1,3}^{*}$ to have

$$
C_{j}\left[\psi_{0}(y)\right] \sim \frac{C_{l, 3}^{*}}{\Gamma\left(-\frac{1}{2}\right)} j^{-\frac{1}{2}} \sim C_{l, 3} j^{-\frac{1}{2}}
$$

where

$$
C_{l, 3}=\frac{C_{l, 3}^{*}}{2 \sqrt{\pi}}
$$

Hence, we have

$$
\pi_{0, \jmath} \sim C_{l, 3} j^{-\frac{3}{2}}\left(\frac{1}{y_{1}}\right)^{j}
$$

which leads to the result of $\pi_{0, j}$ in case (iii). 


\subsection{Exact Tail Asymptotics of $\pi_{i, j}$ for fixed $i$}

To characterize the exact tail asymptotics for $\pi_{i, j}$ with $i \neq 0$, we need to analyze the generating function $\psi_{i}(y)$. To give an expression for $\psi_{i}(y)$, first we give a lemma.

Lemma 5.2 For $\psi_{i}(y)=\sum_{j=0}^{\infty} \pi_{\imath, j} y^{j}$ with $i=1,2, \ldots$, we have the following equations:

$$
\begin{aligned}
y c(y) \psi_{1}(y)+[c(y)+y b(y)] \psi_{0}(y) & =\overline{p q} r(1-y) \pi_{0,0}, \\
c(y) \psi_{i+1}(y)+b(y) \psi_{i}(y)+a(y) \psi_{i-1}(y) & =0 \quad \text { for } i \geq 1,
\end{aligned}
$$

where $a(y), b(y)$ and $c(y)$ are given in (3.34) (3.35) and (3.36), respectively.

Proof. For (5.8), from the transition diagram Fig 2.3, we know $1-p_{0,0}=$ $p_{1,0}+p_{1,1}+p_{0,1}+p_{-1,1}+p_{-1,0}$. Then, we obtain

$$
\begin{aligned}
\left(1-p_{00}\right) \sum_{j=1}^{\infty} \pi_{i, j} y^{j}= & p_{1,0} \sum_{j=1}^{\infty} \pi_{i-1, j} y^{j}+p_{-1,0} \sum_{j=1}^{\infty} \pi_{i+1, j} y^{j}+p_{0,1} \sum_{j=1}^{\infty} \pi_{i, j-1} y^{j} \\
& +p_{1,1} \sum_{j=1}^{\infty} \pi_{i-1, j-1} y^{j}+p_{-1,1} \sum_{j=1}^{\infty} \pi_{i+1, j-1} y^{j}
\end{aligned}
$$

In the above equation, take term $p_{0,1} \sum_{j=1}^{\infty} \pi_{i, j-1} y^{j}$ as an example, it can be simplified to the following,

$$
\begin{aligned}
p_{0,1} \sum_{j=1}^{\infty} \pi_{i, j-1} y^{j} & =p_{0,1}\left(\pi_{i, 0} y+\pi_{i, 1} y^{2}+\pi_{i, 2} y^{3}+\ldots\right) \\
& =p_{0,1} y\left(\pi_{i, 0}+\pi_{i, 1} y+\pi_{i, 2} y^{2}+\ldots\right) \\
& =p_{0,1} y \psi_{i}(y)
\end{aligned}
$$


Using a similar idea, we further simplify the equation,

$$
\begin{aligned}
& \left(1-p_{0,0}\right) \psi_{i}(y)-\left(1-p_{00}\right) \pi_{i, 0} \\
= & p_{1,0} \psi_{i-1}(y)-p_{1,0} \pi_{\imath-1,0}+p_{-1,0} \psi_{i+1}(y)-p_{-1,0} \pi_{i+1,0}+p_{0,1} y \psi_{i}(y) \\
& +p_{1,1} y \psi_{i-1}(y)+p_{-1,1} y \psi_{i+1}(y) \\
& {\left[p_{-1,0}+p_{-1,1} y\right] \psi_{i+1}(y)-\left(1-p_{0,0}\right) \pi_{i, 0} } \\
= & {\left[1-p_{00}-p_{0,1} y\right] \psi_{i}(y)-\left[p_{1,0}+p_{1,1} y\right] \psi_{i-1}(y)-p_{1,0} \pi_{\imath-1,0}-p_{-1,0} \pi_{i+1,0} }
\end{aligned}
$$

Then, from balance equation (2.25) we have

$$
\left(p_{-1,0}+p_{-1,1} y\right) \psi_{i+1}(y)=\left(1-p_{00}-p_{0,1} y\right) \psi_{i}(y)-\left(p_{1,0}+p_{1,1} y\right) \psi_{i-1}(y)
$$

using $a(y), b(y)$, and $c(y)$ given in (3.34), (3.35), and (3.36), respectively, the above equation leads to result (5.8). For (5.7), we start from the fundamental form of generating function,

$$
\begin{aligned}
& P(x, y)=\frac{H_{2}(x, y) \psi_{0}(y)+H_{0}(x, y) \pi_{00}}{-y K_{0}(x, y)}, \\
& \frac{\partial}{\partial x} P(x, y) \\
= & -\frac{1}{y}\left\{\frac{\left[\frac{\partial}{\partial x} H_{2}(x, y) \psi_{0}(y)+\frac{\partial}{\partial x} H_{0}(x, y) \pi_{0.0}\right]}{K_{0}(x, y)}-\frac{\frac{\partial}{\partial x} K_{0}(x, y)\left[H_{2}(x, y) \psi_{0}(y)+H_{0}(x, y) \pi_{0.0}\right]}{\left[K_{0}(x, y)\right]^{2}}\right\} \\
= & -\frac{1}{y}\left\{\frac{\left[\frac{\partial}{\partial x} H_{2}(x, y) \psi_{0}(y)+\frac{\partial}{\partial x} H_{0}(x, y) \pi_{0.0}\right]}{K_{0}(x, y)}-\frac{-y \frac{\partial}{\partial x} K_{0}(x, y)\left[H_{2}(x, y) \psi_{0}(y)+H_{0}(x, y) \pi_{0.0}\right]}{K_{0}(x, y)}\right\} \\
= & -\frac{1}{y}\left\{\frac{\left[\frac{\partial}{\partial x} H_{2}(x, y) \psi_{0}(y)+\frac{\partial}{\partial x} H_{0}(x, y) \pi_{0.0}\right]}{K_{0}(x, y)}-\frac{-y \frac{\partial}{\partial x} K_{0}(x, y)}{K_{0}(x, y)} P(x, y)\right\} \\
= & -\frac{1}{y}\left\{\frac{\left[\frac{\partial}{\partial x} H_{2}(x, y) \psi_{0}(y)+\frac{\partial}{\partial x} H_{0}(x, y) \pi_{0.0}\right]}{K_{0}(x, y)}+\frac{y \frac{\partial}{\partial x} K_{0}(x, y)}{K_{0}(x, y)} P(x, y)\right\} .
\end{aligned}
$$


Using $\frac{\partial}{\partial x} H_{2}(x, y)=c(y), K_{0}(0, y)=c(y),\left.\frac{\partial}{\partial x} K_{0}(x, y)\right|_{x=0}=b(y)$ and $P(0, y)=$ $\frac{H_{2}(0, y) \psi_{0}(y)}{-y K_{0}(0, y)}=\psi_{0}(y)$, we obtain

$$
\begin{aligned}
\psi_{1}(y) & =P_{x}(0, y)=-\frac{1}{y}\left\{\frac{c(y) \psi_{0}(y)+\frac{\partial}{\partial x} H_{0}(x, y) \pi_{00}}{c(y)}+\frac{y b(y) \psi_{0}(y)}{c(y)}\right\} \\
& =\left\{\frac{[c(y)+y b(y)] \psi_{0}(y)+\frac{\partial}{\partial x} H_{0}(x, y) \pi_{00}}{-y c(y)}\right\}
\end{aligned}
$$

which yields result (5.7).

Now with this result, we are ready to express $\psi_{\imath}(y)$. This is given in the following proposition.

Proposition 5.1 For $i \geq 0$,

$$
\psi_{\imath}(y)=\psi_{0}(y)\left(\frac{1}{x_{1}(y)}\right)^{2}
$$

where $y$ is in the cut plane $C_{y}-\left[y_{1}, y_{2}\right]$ and $y \neq \eta_{1}$.

Proof. We know for $|y| \leq 1$, the recursive equation (5.8) has a general solution

$$
\psi_{\imath}(y)=K_{1}\left[r_{1}(y)\right]^{2}+K_{2}\left[r_{2}(y)\right]^{2}
$$

with

$$
\begin{aligned}
& r_{1}(y)=\frac{-b(y)-\sqrt{\Delta(y)}}{2 c(y)}=\frac{2 a(y)}{-b(y)+\sqrt{\Delta(y)}}=\frac{1}{x_{1}(y)}, \\
& r_{2}(y)=\frac{-b(y)+\sqrt{\Delta(y)}}{2 c(y)}=\frac{2 a(y)}{-b(y)-\sqrt{\Delta(y)}}=\frac{1}{x_{2}(y)} .
\end{aligned}
$$


Now, we need to express $K_{1}$ and $K_{2}$. Take $i=1$, we have

$$
\begin{aligned}
\psi_{0}(y) & =K_{1}+K_{2}, \\
y c(y)\left[K_{1} r_{1}(y)+K_{2} r_{2}(y)\right]+[c(y)+y b(y)] \psi_{0}(y) & =\overline{p q} r(1-y) \pi_{0.0} .
\end{aligned}
$$

Express $K_{1}$ in terms of $K_{2}$ to have,

$$
\begin{aligned}
y c(y)\left\{\left[\psi_{0}(y)-K_{2}\right] r_{1}(y)+K_{2} r_{2}(y)\right\}+[c(y)+y b(y)] \psi_{0}(y)= & \overline{p q} r(1-y) \pi_{0.0}, \\
-y c(y) K_{2} r_{1}(y)+y c(y) \psi_{0}(y) r_{1}(y)+y c(y) K_{2} r_{2}(y)= & \overline{p q} r(1-y) \pi_{0.0} \\
& -[c(y)+y b(y)] \psi_{0}(y) .
\end{aligned}
$$

$K_{2}\left\{-y c(y) r_{1}(y)+y c(y) r_{2}(y)\right\}=\overline{p q} r(1-y) \pi_{0.0}-[c(y)+y b(y)] \psi_{0}(y)-y c(y) \psi_{0}(y) r_{1}(y)$,

$$
K_{2} y c(y)\left[r_{2}(y)-r_{1}(y)\right]=\overline{p q} r(1-y) \pi_{0.0}-\psi_{0}(y)\left[c(y)+y b(y)+y c(y) r_{1}(y)\right] .
$$

Using $c(y)\left[r_{2}(y)-r_{1}(y)\right]=c(y) \frac{x_{1}(y)-x_{2}(y)}{x_{1}(y) x_{2}(y)}=\frac{4 a(y) c(y) \sqrt{\Delta(y)}}{b(y)^{2}-\Delta(y)}=\Delta(y), b(y) x_{1}(y)=$ $-a(y) x_{1}^{2}(y)$, and $x_{2}(y) x_{1}(y)=\frac{c(y)}{a(y)}$, do further simplifications,

$$
\begin{aligned}
& K_{2} y \sqrt{\Delta y} \\
= & \overline{p q} r(1-y) \pi_{00}-\psi_{0}(y)\left[c(y)+y b(y)+y c(y) r_{1}(y)\right] \\
= & \overline{p q} r(1-y) \pi_{0.0}-\psi_{0}(y)\left[c(y)+y \frac{b(y) x_{1}(y)+c(y)}{x_{1}(y)}\right. \\
= & \overline{p q} r(1-y) \pi_{0.0}-\psi_{0}(y)\left[c(y)+y \frac{-a(y) x_{1}^{2}(y)}{x_{1}(y)}\right] \\
= & \overline{p q} r(1-y) \pi_{0.0}-\psi_{0}(y)\left[c(y)-y a(y) x_{1}(y)\right],
\end{aligned}
$$




$$
\begin{aligned}
& \overline{p q} r(1-y) \pi_{0.0}-\psi_{0}(y)\left[c(y)-y a(y) x_{1}(y)\right] \\
= & \overline{p q} r(1-y) \pi_{0.0}-\frac{x_{2}(y) \bar{p} \bar{q} r(1-y) \pi_{0,0}}{(q y+\bar{q}) \bar{p} r\left[x_{2}(y)-y\right]}\left[c(y)-y a(y) x_{1}(y)\right] \\
= & \bar{p} r(1-y) \pi_{0.0}-\frac{x_{2}(y) \overline{p q} r(1-y) \pi_{0,0}}{c(y)\left[x_{2}(y)-y\right]}\left[c(y)-y a(y) x_{1}(y)\right] \\
= & \overline{p q} r(1-y) \pi_{0.0}\left\{1-\frac{x_{2}(y)}{c(y)\left[x_{2}(y)-y\right]}\left[c(y)-y a(y) x_{1}(y)\right]\right\} \\
= & \overline{p q} r(1-y) \pi_{0.0}\left\{1-\frac{x_{2}(y) c(y)-y a(y) x_{2}(y) x_{1}(y)}{c(y)\left[x_{2}(y)-y\right]}\right\} \\
= & \overline{p q} r(1-y) \pi_{0.0}\left\{1-\frac{x_{2}(y) c(y)-y a(y) \frac{c(y)}{a(y)}}{c(y)\left[x_{2}(y)-y\right]}\right\} \\
= & \overline{p q} r(1-y) \pi_{0.0}\left\{\frac{c(y)\left[x_{2}(y)-y\right]}{c(y)\left[x_{2}(y)-y\right]}-\frac{x_{2}(y) c(y)-y c(y)}{c(y)\left[x_{2}(y)-y\right]}\right\}=0 .
\end{aligned}
$$

Hence, we have $K_{2}=0$, which yields $K_{1}=\psi_{0}(y)$, then from (5.9),

$$
\psi_{i}(y)=\psi_{0}(y)\left(\frac{1}{x_{1}(y)}\right)^{i}
$$

Since $\psi_{i}(y)$ has positive coefficients for its Taylor expansion at $y=0$ and also $\frac{1}{x_{1}(y)}$ has no poles in the cut plane, then $\psi_{2}(y)$ has the same dominant singular point as $\psi_{0}(y)$. Now we can show the exact tail asymptotics of $\pi_{\imath, j}$ for a fixed $i$ based on $\psi_{2}(y)$ as stated in theorem 1.2.

Theorem 1.2 Proof. For case (i), it follows that,

$$
\begin{aligned}
\lim _{\substack{y \\
\eta_{1}}}\left(1-\frac{y}{\eta_{1}}\right) \psi_{i}(y) & =\lim _{\frac{y}{\eta_{1}} \rightarrow 1}\left(1-\frac{y}{\eta_{1}}\right) \psi_{0}(y)\left(\frac{1}{x_{1}(y)}\right)^{\imath} \\
& =C_{l, 1}\left(\frac{1}{x_{1}\left(\eta_{1}\right)}\right)^{i}=C_{l, 1} \beta^{2}
\end{aligned}
$$

where

$$
\beta=\frac{1}{x_{1}\left(\eta_{1}\right)}=\frac{x_{2}\left(\eta_{1}\right)}{x_{1}\left(\eta_{1}\right) x_{2}\left(\eta_{1}\right)}=\frac{\eta_{1} c\left(\eta_{1}\right)}{a\left(\eta_{1}\right)}=\frac{\eta_{1} \bar{p} r}{p \bar{r}}
$$


In order to get the asymptotics for $\pi_{i, j}$ with fixed $i$, we rewrite $\psi_{i}(y)$ in the following form:

$$
\psi_{i}(y)=\sum_{j=0}^{\infty} \pi_{i, j} y^{j}=\sum_{j=0}^{\infty}\left(\eta_{1}\right)^{j} \pi_{i, j}\left(\frac{y}{\eta_{1}}\right)^{j}=\sum_{j=0}^{\infty} f_{j} z^{j}
$$

where $f_{j}=\left(\eta_{1}\right)^{j} \pi_{i, j}$ and $z=\frac{y}{\eta_{1}}$. It is obvious that for $i \geq 0, \psi_{i}(y)$ is analytic in the region $\Delta(\phi, \epsilon)=\left\{y:\left|\frac{y}{\eta_{1}}\right| \leq 1+\epsilon,\left|\operatorname{Arg}\left(\frac{y}{\eta_{1}}-1\right)\right| \geq \phi, \epsilon>0,0<\phi<\pi / 2\right\}$ except at $\frac{y}{\eta_{1}}=1$. Then we can use Theorem 4.1 on $\psi_{i}(y)$ with $s=-1$ and $K=C_{l, 1} \beta^{i}$ to have

$$
C_{j}\left[\psi_{i}(y)\right] \sim \frac{C_{l, 1} \beta^{i}}{\Gamma(1)} j^{1-1} \sim C_{l, 1} \beta^{i}
$$

Hence, we have

$$
\pi_{i, j} \sim C_{l, 1} \beta^{i}\left(\frac{1}{\eta_{1}}\right)^{j}
$$

which leads to the result of $\pi_{\imath, j}$ in case (i).

For case (ii), it follows that

$$
\begin{aligned}
& \lim _{\frac{y}{y_{1}} \rightarrow 1} \sqrt{1-\frac{y}{y_{1}}} \psi_{i}(y) \\
= & \lim _{\frac{y}{y_{1}} \rightarrow 1} \sqrt{1-\frac{y}{y_{1}}} \psi_{0}(y)\left(\frac{1}{x_{1}(y)}\right)^{i} \\
= & C_{l, 2}^{*}\left(\frac{1}{x_{1}\left(y_{1}\right)}\right) \beta^{i},
\end{aligned}
$$

where

$$
\beta=\frac{1}{x_{1}\left(y_{1}\right)}=\sqrt{\frac{a\left(y_{1}\right)}{c\left(y_{1}\right)}}=\sqrt{\frac{p \bar{r}}{\bar{p} r}}
$$


Similarly to case (i), we rewrite $\psi_{i}(y)$,

$$
\psi_{i}(y)=\sum_{j=0}^{\infty} \pi_{i, j} y^{j}=\sum_{j=0}^{\infty}\left(y_{1}\right)^{j} \pi_{i, j}\left(\frac{y}{y_{1}}\right)^{j}=\sum_{j=0}^{\infty} f_{j} z^{j}
$$

where $f_{j}=\left(y_{1}\right)^{j} \pi_{i, j}$ and $z=\frac{y}{y_{1}}$. It is obvious that for $i \geq 0, \psi_{i}(y)$ is analytic in the region $\Delta(\phi, \epsilon)=\left\{y:\left|\frac{y}{y_{1}}\right| \leq 1+\epsilon,\left|\operatorname{Arg}\left(\frac{y}{y_{1}}-1\right)\right| \geq \phi, \epsilon>0,0<\phi<\pi / 2\right\}$ except at $\frac{y}{y_{1}}=1$. Then use Theorem 4.1 on $\psi_{\imath}(y)$ with $s=-\frac{1}{2}$ and $K=C_{1,2}^{*} \beta^{i}$ to have

$$
C_{j}\left[\psi_{i}(y)\right] \sim \frac{C_{l, 2}^{*} \beta^{i}}{\Gamma\left(\frac{1}{2}\right)} j^{-\frac{1}{2}} \sim C_{l, 2} \beta^{i} j^{-\frac{1}{2}}
$$

Hence, we have

$$
\pi_{i, j} \sim C_{l, 2} \beta^{i} j^{-\frac{1}{2}}\left(\frac{1}{y_{1}}\right)^{j}
$$

which leads to the result of $\pi_{i, j}$ in case ii).

For case (iii),

$$
\begin{aligned}
\psi_{i}^{\prime}(y)= & \psi_{0}^{\prime}(y)\left(\frac{1}{x_{1}(y)}\right)^{i}-\psi_{0}(y) i\left(\frac{1}{x_{1}(y)}\right)^{i-1} \frac{d x_{1}(y)}{d y} \\
= & \psi_{0}^{\prime}(y)\left(\frac{1}{x_{1}(y)}\right)^{i}-\psi_{0}(y) i\left(\frac{1}{x_{1}(y)}\right)^{i-1} \\
& \left\{\frac{a(y)\left[-b^{\prime}(y)+\frac{b(y) b^{\prime}(y)-2 a(y) c^{\prime}(y)-2 a^{\prime}(y) c(y)}{\sqrt{d\left(1-\frac{y}{y_{1}}\right)\left(1-\frac{y}{y_{1}}\right)}}\right]-a^{\prime}(y)[-b(y)-\sqrt{\Delta y}]}{2[a(y)]^{2}}\right\} .
\end{aligned}
$$


Note that $\lim _{\frac{y}{y_{1}} \rightarrow 1} \sqrt{1-\frac{y}{y_{1}}}\left\{-a(y) b^{\prime}(y)-a^{\prime}(y)[--b(y)-\sqrt{\Delta y}]\right\}=0$ since $\left\{-a(y) b^{\prime}(y)-a^{\prime}(y)[-b(y)-\sqrt{\Delta y}]\right\}$ is continuous at $y_{1}$, we obtain

$$
\begin{aligned}
& \lim _{\frac{y}{y_{1}} \rightarrow 1} \sqrt{1-\frac{y}{y_{1}}} \psi_{i}^{\prime}(y) \\
= & \lim _{\frac{y}{y_{1}} \rightarrow 1} \sqrt{1-\frac{y}{y_{1}}} \psi_{0}^{\prime}(y)\left(\frac{1}{x_{1}(y)}\right)^{i} \\
& -\lim _{\frac{y}{y_{1}} \rightarrow 1} \psi_{0}(y) i\left(\frac{1}{x_{1}(y)}\right)^{i-1} \frac{\sqrt{1-\frac{y}{y_{1}}}\left[b(y) b^{\prime}(y)-2 a(y) c^{\prime}(y)-2 a^{\prime}(y) c(y)\right]}{\sqrt{d\left(1-\frac{y}{y_{1}}\right)\left(1-\frac{y}{y_{2}}\right)}} \\
= & C_{l, 3}^{*}\left(\frac{1}{x_{1}\left(y_{1}\right)}\right)^{i}-\psi_{0}\left(y_{1}\right) i\left(\frac{1}{x_{1}\left(y_{1}\right)}\right)^{i-1} \frac{b\left(y_{1}\right) b^{\prime}\left(y_{1}\right)-2 a\left(y_{1}\right) c^{\prime}\left(y_{1}\right)-2 a^{\prime}\left(y_{1}\right) c\left(y_{1}\right)}{\sqrt{d\left(1-\frac{y_{1}}{y_{2}}\right)}} \\
= & C_{l, 3}^{*}\left(\frac{1}{x_{1}\left(y_{1}\right)}\right)^{i}[1+H i] \\
= & C_{l, 3}^{*} \beta^{i}[1+H i],
\end{aligned}
$$

where

$$
\begin{aligned}
\beta & =\frac{1}{x_{1}\left(y_{1}\right)}=\sqrt{\frac{p \bar{p}}{\bar{p} r}}, \\
H & =-\frac{\psi_{0}\left(y_{1}\right) x_{1}\left(y_{1}\right)}{C_{l, 3}^{*}}\left[\frac{b\left(y_{1}\right) b^{\prime}\left(y_{1}\right)-2 a\left(y_{1}\right) c^{\prime}\left(y_{1}\right)-2 a^{\prime}\left(y_{1}\right) c\left(y_{1}\right)}{\sqrt{d\left(1-\frac{y_{1}}{y_{2}}\right)}}\right] \\
& =-\frac{\psi_{0}\left(y_{1}\right) x_{1}\left(y_{1}\right)}{C_{l, 3}^{*}}\left[\frac{q\left[(\overline{p r}-p r)^{2}\left(q y_{1}+\bar{q}\right)-(\overline{p r}+p r)\right]}{\sqrt{d\left(1-\frac{y_{1}}{y_{2}}\right)}}\right] .
\end{aligned}
$$

To find the asympototics of $\pi_{i, j}$, we rewrite $\psi_{i}^{\prime}(y)$,

$$
\psi_{\imath}^{\prime}(y)=\sum_{j=0}^{\infty} j \pi_{i, j} y^{j-1}=\sum_{j=0}^{\infty}(j+1) \pi_{i, j+1} y_{1}^{j}\left(\frac{y}{y_{1}}\right)^{\jmath}=\sum_{j=0}^{\infty} f_{\jmath+1} z^{\jmath}
$$

where $f_{j+1}=(j+1) \pi_{i, j+1} y_{1}^{j}$ and $z=\frac{y}{y_{1}}$. It is obvious that for $i \geq 0, \psi_{i}(y)$ is analytic in the region $\Delta(\phi, \epsilon)=\left\{y:\left|\frac{y}{y_{1}}\right| \leq 1+\epsilon,\left|\operatorname{Arg}\left(\frac{y}{y_{1}}-1\right)\right| \geq \phi, \epsilon>0,0<\right.$ 
$\phi<\pi / 2\}$ except at $\frac{y}{y_{1}}=1$. Then use Lemma 4.1 on $\psi_{2}^{\prime}(y)$ with $s=-\frac{1}{2}$ and $K=C_{1,3}^{*} \beta^{i}[1+H i]$ to have

$$
C_{j}\left[\psi_{i}^{\prime}(y)\right] \sim \frac{C_{l, 3}^{*} \beta^{i}(1+H i)}{\Gamma\left(-\frac{1}{2}\right)} j^{-\frac{1}{2}} \sim C_{l, 3} \beta^{2}(1+H i) j^{-\frac{1}{2}}
$$

Hence, we have

$$
\pi_{i, \jmath} \sim C_{l, 3} \beta^{i}(1+H i) j^{-\frac{3}{2}}\left(\frac{1}{y_{1}}\right)^{j}
$$

which leads to the result of $\pi_{i, j}$ in case (iii). 


\section{Chapter 6}

\section{Exact Tail Asymptotics for the High-Priority Queue}

In this chapter, we establish the result shown in Theorem 1.1. To characterize the tail asymptotics in $\pi_{i, j}$ for a fixed $j \geq 0$, we analyze the generating function of $\varphi_{j}(x)$ given in (3.52).

Lemma 6.1 For $j \geq 0$,

$$
\varphi_{j}(x) \sim \frac{C \pi_{0,0}}{\left(1-r_{0} x\right)^{j+1}}, \text { as } r_{0} x \rightarrow 1
$$

with $\pi_{0,0}$ given in (3.31) and

$$
C=-\frac{1}{2 p q r \overline{p q r}}\left[(\overline{p r}+p r) \bar{q}-1+\sqrt{[(\overline{p r}-p r) \bar{q}-1]^{2}-4 p r \overline{p r q}^{2}}\right]
$$

Proof. For case $j=0$,

$$
\varphi_{0}(x)=\frac{\pi_{0,0}}{1-r_{0} x}
$$


This is proved in a previous section. Hence it is true for $j=0$. We know the following,

$$
\varphi_{\jmath}(x)=\frac{a_{\jmath}}{1-r_{0} x}+\frac{r_{0}\left[p_{0,1}+p_{1,1}\left(x+x_{2}\right)\right]}{p_{1,0}\left(1-r_{0} x\right)}\left(\varphi_{\jmath-1}(x)-\varphi_{\jmath-1}\left(x_{2}\right)\right) .
$$

For $j=1$,

$$
\begin{aligned}
\varphi_{1}(x) & =\frac{a_{1}}{1-r_{0} x}+\frac{r_{0}\left[p_{0,1}+p_{1,1}\left(x+x_{2}\right)\right]}{p_{1,0}\left(1-r_{0} x\right)}\left(\varphi_{0}(x)-\varphi_{0}\left(x_{2}\right)\right), \\
\left(1-r_{0} x\right)^{2} \varphi_{1}(x) & =a_{1}\left(1-r_{0} x\right)+\frac{r_{0}\left(p_{0,1}+p_{1,1}\left(x+x_{2}\right)\right)}{p_{1,0}}\left(\varphi_{0}(x)\left(1-r_{0} x\right)-\varphi_{0}\left(x_{2}\right)\left(1-r_{0} x\right)\right) .
\end{aligned}
$$

Since $a_{1}$ and $\varphi_{0}\left(x_{2}\right)$ are both constants, when we take limit as $r_{0} x \rightarrow 1$, both of these two terms go to zeros. We have,

$$
\lim _{r_{0} x \rightarrow 1}\left(1-r_{0} x\right)^{2} \varphi_{1}(x)=C \pi_{0,0}
$$

The constant $C$ is obtained from the following steps:

$$
\begin{aligned}
C & =\lim _{r_{0} x \rightarrow 1} \frac{r_{0}\left(p_{0,1}+p_{1,1}\left(x+x_{2}\right)\right)}{p_{1,0}} \\
& =\frac{r_{0}}{p_{1,0}}\left(p_{0,1}+p_{1,1}\left(1 / r_{0}+x_{2}\right)\right) \\
& =\frac{r_{0}}{p_{1,0}}\left(p_{0,1}+p_{1,1}\left(x_{1}+x_{2}\right)\right) \\
& =\frac{1}{x_{1} p \overline{\overline{q r}}}[(\overline{p r}+p r) q+1-(\overline{p r}+p r) \bar{q}] \\
& =-\frac{b(0)+\sqrt{\Delta(0)}}{2 c(0)} \frac{1}{p \overline{q r}} \\
& =-\frac{1}{2 p q r \overline{p q r}}\left[(\overline{p r}+p r) \bar{q}-1+\sqrt{[(\overline{p r}-p r) \bar{q}-1]^{2}-4 p r \overline{p r q}^{2}}\right] .
\end{aligned}
$$


We use induction to continue the proof. Assume that this is true for $j=k$, or

$$
\lim _{r_{0} x \rightarrow 1}\left(1-r_{0} x\right)^{k+1} \varphi_{k}(x)=C \pi_{0,0}
$$

we need to verify this is also true for case $j=k+1$.

$$
\begin{aligned}
\varphi_{k+1}(x)= & \frac{a_{k+1}}{1-r_{0} x}+\frac{r_{0}\left[p_{0,1}+p_{1,1}\left(x+x_{2}\right)\right]}{p_{1,0}\left(1-r_{0} x\right)}\left[\varphi_{k}(x)-\varphi_{k}\left(x_{2}\right)\right], \\
\left(1-r_{0} x\right)^{k+2} \varphi_{k+1}(x)= & a_{k+1}\left(1-r_{0} x\right)^{k+1} \\
& +\frac{r_{0}\left[p_{0,1}+p_{1,1}\left(x+x_{2}\right)\right]}{p_{1,0}}\left[\varphi_{k}(x)\left(1-r_{0} x\right)^{k+1}-\varphi_{k}\left(x_{2}\right)\left(1-r_{0} x\right)^{k+1}\right], \\
\lim _{r_{0} x \rightarrow 1}\left(1-r_{0} x\right)^{k+2} \varphi_{k+1}(x)= & \lim _{r_{0} x \rightarrow 1} C \varphi_{k}(x)\left(1-r_{0} x\right)^{k+1}, \\
= & C \pi_{0,0} \quad[\text { using 6.3]. }
\end{aligned}
$$

This is equivalent to the previous case for $j=k$. This completes the proof of the lemma.

Theorem 1.1 Proof. It is obvious that for $j \geq 0, \varphi_{\jmath}(x)$ is analytic in the region $\Delta(\phi, \epsilon)=\left\{x:\left|r_{0} x\right| \leq 1+\epsilon,\left|\operatorname{Arg}\left(r_{0} x-1\right)\right| \geq \phi, \epsilon>0,0<\phi<\pi / 2\right\}$, except at $r_{0} x=1$. Using the Tauberian theorem, the coefficients of $\pi_{n, 3}$ in the Taylor expression or $\varphi_{\jmath}(x)$ satisfy

$$
\begin{aligned}
\frac{C_{n}\left[\varphi_{\jmath}(x)\right]}{r_{0}^{n}} & \sim C \pi_{0,0} \frac{1}{\Gamma(j+1)} n^{(\jmath+1)-1}, \\
& \sim C \pi_{0,0} \frac{n^{3}}{j !}, \quad j \geq 0 .
\end{aligned}
$$

Hence, we have

$$
\pi_{n, \jmath} \sim C \pi_{0,0} \frac{1}{\jmath !} n^{\jmath} r_{0}^{n}, \quad \jmath \geq 0
$$


This completes the proof for tail asymptotics along the high-priority queue direction with a fixed number of low-priority customers. 


\section{Chapter 7}

\section{Conclusion}

In this thesis, we considered a discrete-time preemptive priority queue model with two classes of customers and a single server. The two classes of customers have asymmetric arrival probability and symmetric service time. Compared to the classical continuous-time priority queue, the discrete-time priority queue can be viewed as a counter-part case and the system model is more complicated. We used the generating function approach to characterize the exact tail asymptotics for the joint stationary distributions since the generating function involved can be expressed explicitly. We have concluded that there are only three types of exact tail asymptotics along the low-priority queue direction, which are: exact geometric, geometric multiplied by a power function with power $-1 / 2$ or $-3 / 2$. We also provided the regions for each of the three types of tail asymptotics. Along the high-priority direction, the tail asymptotics is different from these three types. The results are parallel to the continuous-time priority queue results.

We have only considered the tail asymptotics for the joint stationary distribution. Some future research on this model could be rewarding:

1. Characterization of the exact tail asymptotics in the marginal stationary 
distributions $\pi_{j}^{(l)}$ along the low-priority direction. This may be worked based on the existing generating function and computing $P(1, y)$.

2. Tail asymptotics in the stationary distribution $\pi_{n}^{T}$ of the total number of customers in the system needs to be developed. Based on the existing generating function and some computations of $P(x, x)$, this may be possible to complete.

3. Discrete-time preemptive priority queue with asymmetric service probabilities is interesting to study in the next step.

4. Exact tail asymptotics for this discrete-time priority queue model with a bulk arrival probabilities and bulk service probabilities could be considered for future research 


\section{Bibliography}

[1] Adan, I., Foley, R.D. and McDonald, D.R.: Exact asymptotics for the stationary distribution of a Markov chain: a production modle, Queueing Syst. 62, 311-344 (2009).

[2] Alfa, A.S.: Matrix-geometric solution of discrete time MAP/PH/1 priority queue. Nav. Res. Logist. 45, 23-50 (1998).

[3] Alfa, A.S. and Liu, B., He, Q.-M.: Discrete-time analysis of MAP/PH/1 multiclass general preemptive priority queue. Nav. Res. Logist. 50, 662-682 (2003).

[4] Bender, E.: Asymptotics methods in enumeration. SIAM Rev. 16, 485-513 (1974).

[5] Borovkov, A.A. and Mogul'skii, A.A.: Large deviations for Markov chains in the positive quadrant. Russ. Math. Surv. 56, 803-916 (2001).

[6] Bousquet-Melou, M.: Walks in the quarter plane: Kreweras' algebraic model. Ann. Appl. Probab. 15, 1451-1491 (2005).

[7] Cohen, J.W. and Boxma, O.J.: Boundary Value Problems in Queueing System Analysis. North-Holland, Amsterdam (1983). 
[8] Drekic, S. and Woolford, D.G.: A preemptive priority queue with balking. Eur. J. Oper. Res. 164, 387-401 (2005).

[9] Fayolle, G. and Iasnogorodski, R.: Two coupled processors: the reduction to a Remann-Hilbert problem. Z. Wahrscheinlichkeitstheor. Verw. Geb. 47, 325-352 (1979).

[10] Fayolle, G., Iasnogorodski, R. and Malyshev, V.: Random Walks in the Quarter-Plane. Springer, New York (1991).

[11] Fayolle, G., King P.J.B. and Mitrani, I.: The solution of certain twodimensional Markov models, Adv. Appl. Probab. 14, 295-308 (1982).

[12] Flatto, L.: Two parallel queues created by arrivals wit two demands II SIAM J.APPL. Math. 45, 861-878 (1985).

[13] Flatto, L. and Hahn, S.: Two parallel queues created by arrivals with two demands I. SIAM J. Appl. Math. 44, 1041-1053 (1984).

[14] Flatto, L. and McKean, H.P.: Two queues in parallel. Commun. Pure Appl. Math. 30, 255-263 (1977).

[15] Flajolet, P. and Odlyzko, A.: Singularity analysis of generating function. SIAM J. Discrete Math. 3. 216-240 (1990).

[16] Foley, R.D. and McDonald, R.D.: Join the shortest queue: stability and exact asymptotics. Ann. Appl. Probab. 11, 569-607 (2001).

[17] Foley, R.D. and McDonald, R.D.: Large deviation of a modified Jackson network: stability and rough asymptotics. Ann. Appl. Proba. 15, 519-541 (2005). 
[18] Foley, R.D. and McDonald, R.D.: Bridges and networks: exact asymptotics. Ann. Appl. Probab. 15, 542-586 (2005).

[19] Gail, H.R. and Hantler, S.L., Taylor, B.A.: Analysis of a non-preemptive priority multiserver queue. Adv. Appl. Probab. 20, 852-879 (1988).

[20] Gail, H.R. and Hantler, S.L., Taylor, B.A.: On preemptive Markovian queue with multiple servers and two priority classes. Math. Oper. Res. 17, 365-391 (1992).

[21] Guillemin, F. and van Leeuwarden, J.: Rare event asymptotics for a random walk in the quarter plane. To appear in Queueing Syst. (2010).

[22] Haque, L.: Tail behaviour for stationary distribution for two-dimensional stochastic models. Ph.D. Thesis, Carleton University, Ottawa, ON, Canada (2003).

[23] Haque, L., Liu, L. and Zhao, Y.Q.: Sufficient conditions for a geometric tail in a QBD process with countably many levels and phases. Stoch. Modeis 21(1), 77-99 (2005).

[24] He, Q., Li, H. and Zhao, Y.Q.: Light-tailed behaviour in QBD process with countably many phases. Stoch. Models 25, 50-75 (2009).

[25] Isotupa, K.P.S. and Stanford, D.A.: An infinite-phase quasi-birth-and-death model for the non-preemptive priority $\mathrm{M} / \mathrm{M} / \mathrm{PH} / 1$ queue. Stoch. Models 18, $378-410(2002)$.

[26] Hunter, J.J.: Mathematical techniques of applied probability. Academic Press. New York. (1983). 
[27] Kao, E.P.C. and Narayanan, K.S.: Computing steady-state probabilities of a non-preemptive priority multiserver queue. ORSA J.Comput. 2, 211-218 (1990).

[28] Kroese, D.P., Scheinhardt, W.R.W. and Taylor, P.G.: Spectral properties of the tandem Jackson network, seen as a quasi-birth-and-death process. Ann. Appl. Probab. 14(4), 2057-2089 (2004).

[29] Kurkova, I.A. and Suhov, Y.M.: Malyshev's theory and JS-queues. Asymptotics of stationary probabilities. Ann. Appl. Probab. 13, 1313-1354 (2003).

[30] Li, L., Miyazawa, M. and Zhao, Y.: Geometric decay in a QBD process with countable background states with applications to a join-the-shortest-queue model. Stoch. Models 23, 413-438 (2007).

[31] Li, H. and Zhao, Y.: Exact tail asymptotics in a priority queue - characterizations of the preemptive model, Queueing Syst. 63, 355-381 (2009).

[32] Maertens, T., Walraevens, J. and Bruneel, H.: Priority queueing systems: from probability generating functions to tail probabilities. Queueing Syst. 55, 27-39 (2007).

[33] Malyshev, V.A.: An analytical method in the theory of two-dimensional positive random walks, Sib. Math. J. 13, 1314-1329 (1972).

[34] Malyshev, V.A.: Asymptotic behaviour of stationary probabilities for twodimensional positive random walk. Sib. Math. J. 14, 156-169 (1973).

[35] McDonald, D.R.: Asymptotics of first passage times for random walk in an orthant. Ann. Appl. Probab. 9, 110-145 (1999). 
[36] Miller, D.R.: Computatıonof steady-state probabilities for $M / M / 1$ priority queues. Oper. Res. 29(5), 945-958 (1981).

[37] Mishna, M.: Classifying lattice walks restricted to the quarter plane. J. Comb. Theory Ser. A 116, 460-477 (2009).

[38] Miyazawa, M.: The Markov renewal approarh to $M / G / 1$ type queues with countably many background states. Queueing Syst. 46, 177-196 (2004).

[39] Miyazawa, M.: Doubly QBD process and a solution to the tail decay rate problem. In: Proceedings of the Second Asia-Pacific Symposium on Queueing Theory and Network Applications, Kobe, Japan (2007).

[40] Miyazawa, M.: Two sided DQBD process and solutions to the tail decay rate problem and their applications tothe generalized join shortest queue. In: Yue, W., Takahashi, Y., Takagi, H. (eds.) Advances in Queueing Theory and Network Applications, pp. 3-33, Springer, New York (2009).

[41] Miyazawa, M.: Tail decay rates in double QBD processes and related reflected radom walks. Math. Oper. Res. 34(3), 547--575 (2009).

[42] Miyazawa, M. and Zhao, Y.Q.: The stationary tail asymptotics in the GI/G/1 type queue with countably many background states. Adv. Appl. Probab. 36(4), 1231-1251 (2004).

[43] Morrison, J.A.: Processor sharing for two queues with vastly different rates. Queueing Syst. 57, 19-28 (2007).

[44] Motyer, A.J. and Taylor, P.G.: Decay rates for quasi-birth-and-death processes with countably many phases and tri-diagonal block generators. Ad. Appl. Probab. 38, 522-544 (2006). 
[45] Sleptchenko, A., Adan, I.J.B.F. and van Houtum, G.J.: Joint queue length distribution of multi-class, single-server queues with preemptive priorities. Preprint (2004).

[46] Takahashi, Y., Fujimoto, K. and Makimoto, N.: Geometric decay of the steady-state probabilities in an quasi-birth-and-death process with a countable number of phases. Stoch. Models 17(1), 1-24 (2001).

[47] Takine, T.: Anonpreemptive priority MAP/G/1 queue with two classes of customers. J. Oper. Res. Soc. Jpn. 39, 266-290 (1996).

[48] Wright, P.: Two parallel processors with coupled inputs. Adv. Appl. Probab 24, 986-1007 (1992).

[49] Zhao, J.-A, Li, B., Cao, X.-R. and Ahmad, I: A matrix-analytic solution for the DBMAP/PH/1 priority queue. Queueing Syst 53, 127-145 (2006). 